This item was submitted to Loughborough's Research Repository by the author.

Items in Figshare are protected by copyright, with all rights reserved, unless otherwise indicated.

\title{
Rossby solitary waves in the presence of a critical layer
}

PLEASE CITE THE PUBLISHED VERSION

LICENCE

CC BY-NC-ND 4.0

\section{REPOSITORY RECORD}

Caillol, P., and Roger H.J. Grimshaw. 2019. "Rossby Solitary Waves in the Presence of a Critical Layer". figshare. https://hdl.handle.net/2134/2292. 


\title{
ROSSBY SOLITARY WAVES IN THE PRESENCE OF A CRITICAL LAYER
}

\author{
P. Caillol and R. H. Grimshaw
}

Department of Mathematical Sciences, Loughborough University, UK

\begin{abstract}
This study considers the evolution of weakly nonlinear long Rossby waves in a horizontally sheared zonal current. We consider a stable flow so that the nonlinear time scale is long. These assumptions enable the flow to organize itself into a large-scale coherent structure in the régime where a competition sets in between weak nonlinearity and weak dispersion. This balance is often described by a Korteweg-de-Vries equation. The traditional assumption of a weak amplitude breaks down when the wave speed equals the mean flow velocity at a certain latitude, due to the appearance of a singularity in the leading order equation, which strongly modifies the flow in a critical layer. Here, nonlinear effects are invoked to resolve this singularity, since the relevant geophysical flows have high Reynolds numbers. Viscosity is introduced in order to render the nonlinear critical layer solution unique, but the inviscid limit is eventually taken. By the method of matched asymptotic expansions, this inner flow is matched at the edges of the critical layer with the outer flow. We will show that the critical-layer induced flow leads to a strong rearrangement of the related streamlines and consequently of the potential vorticity contours, particularly in the neighbourhood of the separatrices between the open and closed streamlines. The symmetry of the critical layer vis-à-vis the critical level is also broken. This theory is relevant for the phenomenon of Rossby wave breaking and eventual saturation into a nonlinear wave. Spatially localized solutions are described by a Korteweg-de-Vries equation, modified by new nonlinear terms; depending on the critical-layer shape, this leads to depression or elevation waves. The additional terms are made necessary at a certain order of the asymptotic expansion while matching the inner flow on the dividing streamlines. The new evolution equation supports a family of solitary waves. In this paper we describe in detail the case of a depression wave, and postpone for further discussion the more complex case of an elevation wave.
\end{abstract}

\section{Introduction}

Rossby waves are common features of geophysical flows and can be observed in global weather maps in the mid-latitudes of both hemispheres (Wang et al. (1998)). They make important contributions to nonlinear geophysical dynamics in various ways. Synoptic eddies in the oceans can be modeled by the so-called Rossby solitons, or as barotropic solitary eddies, or as modons, that exist due to a balance between nonlinearity and dispersion due to the Earth's rotation meridional gradient ( $\beta$-effect)(Kizner (1997)). The vortex pair blocking observed in the atmosphere can be modeled as a stationary barotropic (Swaters (1986)) or baroclinic (Malguzzi and Malanote-Rizzoli (1985)) solitary Rossby wave, or as an envelope solitary Rossby wave (Luo (1996)). The long-lived nature of these nonlinear waves depends on the persistence of zonal shearing motions, and is clearly linked to the large-scale coherent features of geophysical flows. One of the most famous motivations related to the study of nonlinear Rossby wave stems from the suggestion by Maxworthy and Redekopp (1976) that the Great Red Spot of Jupiter, and some other features of the Jovian atmosphere, may be a manifestation of planetary solitary waves.

The theory of the stability of shear flows has been the object of an intense study for the past century. However, in general, little can be done analytically if the flow is initially unstable, since then nonlinear terms take importance on a short time scale. The number of unstable modes grows so quickly that it becomes rapidly impossible to describe the motion in a simple way. We here therefore restrict attention to a stable flow so that the nonlinear time scale is long. We consider weakly nonlinear Rossby waves whose meridional wavelength is large compared to the latitudinal extent of the shear layer. These assumptions will enable the flow to organize itself into a long-lived and large-scale coherent structure.

We consider the superposition of a small-amplitude linear Rossby wave on a mean shear flow for which the wave speed equals the mean-flow velocity at a certain latitude. A critical-layer singularity then occurs in the linearized stability analysis, and we examine the subsequent modifications of the flow. The dynamical processes involved within the critical layer can be expected to play an important rôle in the large-scale dynamics of the atmosphere and the oceans. Rossby-wave breaking generates highly inhomogeneous flows, that is, narrow zones, oriented east-west, which are essentially nonlinear whereas one can observe a wave-like motion outside (Bradshaw et al. (2002)). Breaking is also characterized by a rearrangement of the potential vorticity $(\mathrm{PV})$ contours in a more or less irreversible way (McIntyre and 
Palmer (1985)). Nonlinear critical layer theory is attractive for the modeling of Rossby wave breaking because it implies an analysis of two such coupled regions: an inner flow where nonlinear dynamics plays an outstanding rôle and an outer flow where linear motions prevail. Here, we will focus on outer flows which lead to solitary waves. Matching the inner and outer flows leads to the distortion of the PV contours within the critical layer, and yields the characteristic cat's eyes motions: bounded flows where the streamlines are closed. The study of solitary Rossby waves propagating in a horizontally sheared zonal flow was first undertaken by Long (1964), Benney (1966) and Redekopp (1977). These works showed that the amplitude of long Rossby waves in a shear flow obeyed a Korteweg-de Vries (KdV) equation. Here we expect to obtain a KdV equation, but altered by new nonlinear terms. The evolution of a solitary wave may be complicated in this case. However, in many previous studies, solitary waves have been considered in systems whose dynamics is modeled by a modified integrable equation. We only report a few of them which are listed in Kivshar and Malomed (1989) and the many references therein: scattering of a shallow water KdV soliton by a depth inhomogeneity, an ion acoustic soliton in an inhomogeneous plasma and acceleration of Langmuir soliton under the action of nonlinear Landau damping.

In the literature related to shear flows on the $\beta$-plane, Brown and Stewartson (1979) also explored the evolution of a marginal disturbance. But, they only considered an initially regular neutral mode. A critical layer analysis is then not required at leading order, but is postponed to the following order, which is mathematically more tractable since the dynamics is weakly nonlinear. The generalization of Rayleigh's inflection point theorem states that $\beta-U^{\prime \prime}(y)$ must change sign at some value of $y$ for instability to occur. As a result, neutral modes are regular. Singular neutral modes can be found in the stable linear régime: they are subcritical modes. The $\beta$-parameter must be large enough to stabilize the flow. Reynolds stress arguments rule out the existence of singular neutral modes in the linear theory. Nevertheless, singular solutions of the Rayleigh equation exist with a nonlinear critical layer. This is caused by the absence of a phase jump through the critical layer (Benney and Bergeron (1969)). Maslowe and Clarke (2002) generated a singular neutral mode as the result of a resonant interaction between two regular modes. These interactions occur more easily than for the usual resonant wave triad. However, the dynamics does not remain weakly nonlinear as time proceeds (Vanneste (1998)). The two Rossby waves generate an effective forcing which creates a nonlinear critical layer, affecting, in turn their own dynamics. The motion inside the critical layer is characterized by fast oscillations associated with the presence of Rossby waves. Nevertheless, the motion remains essentially linear outside the critical layer as the forcing there is very weak (proportional to the product of the amplitudes of the resonant waves).

Warn and Warn (1978) studied a directly forced critical layer, with a forcing amplitude of $\epsilon$. They showed that when $t=O\left(\epsilon^{-\frac{1}{2}}\right)$ the nonlinear terms become of the same magnitude as the linear terms within the critical layer, whose thickness has decreased to $O\left(\epsilon^{\frac{1}{2}}\right)$. They therefore focused on the nonlinear evolution of the critical layer by using the slow time $T=\epsilon^{\frac{1}{2}} t$, with the initial conditions being the asymptotic state $t \rightarrow \infty$ of the original initial-value problem. They observed that the phase shift strongly evolved with time while no steady state was attained for large $T$. Numerical instabilities prevented the integration of the equations of motion of the critical layer from proceeding. The asymptotic case $k \rightarrow 0$ was analysed and revealed strong time-dependance of the motion within the cat's eyes. But numerical resolution limited the large-scale assumption and noise rapidly appeared for $T=O(1)$. The presence of harmonics of same order as the fundamental was detected outside the critical layer whereas Benney and Bergeron had found this result only inside the critical layer. In the inviscid and long-wave assumptions, Stewartson (1978) found an analytical solution for the motion within the critical layer. Later, Killworth and McIntyre (1985) demonstrated the linear shear instability of this flow with respect to two-dimensional disturbances. The fastest growing disturbances develop more rapidly than the nonlinear evolution time of the critical layer. Haynes (1985) numerically followed up the growth and nonlinear saturation of the initial perturbations. The distribution of vorticity is finally deeply rearranged affecting the absorption properties of the layer. When the wavelength is not so long, instability is not so important (Haynes (1989)). The time-integrated absorptivity may be considerably increased, sometimes to three and four times that predicted by the Stewartson solution. A steady state does not seem to be attained even after a long integration, in agreement with Warn and Warn (1978). The motion within the critical layer is disordered and looks turbulent even when a small dissipation is added (Haynes (1989)).

Our study is distinct from these due to the limiting processes involved. Warn and Warn had chosen: $\nu \rightarrow 0, t \rightarrow \infty$ and $k \rightarrow 0$ whereas we will take: $k \rightarrow 0, t \rightarrow \infty$ and $\nu \rightarrow 0$. With such limit ordering, Redekopp (1977) studied both regular and singular neutral modes but he omitted to the coupling through the streamwise-velocity jump between the critical layer and the outer flow and stopped his expansion at 
the leading order. As a result, only the usual KdV equation emerged.

We study here the evolution of a free singular neutral mode for long space and time scales, both outside and inside the critical layer. Some specific assumptions must be taken concerning the mean flow profile. Indeed, critical layers do not appear on stable monotone profiles with smooth initial conditions in a free problem (Brunet and Warn (1990)). However, these restrictions are not stringent. The evolution of an initial free disturbance to a marginally stable shear flow depends on the scale over which it is made. A sorting out process intervenes controlled by linear equations at the end of which most components of the disturbance are decaying algebraically with time leaving only a mode with a large wavelength (Stewartson (1981)).

In this study the singularity is removed in the critical layer by reintroducing advection terms. The equations of motion are analytically integrated at each order of an asymptotic expansion. Integration constants are determined by using an averaging technique on the viscous components which is a generalization by Redekopp (1977) to a solitary wave of the work of B enney and Bergeron (1969). An additional term $\mathcal{C}_{X}[A]$ appears in the $\mathrm{KdV}$ equation for the modal amplitude $A$, which then has the form,

$$
\partial_{T} A+R_{0} A \partial_{X} A+S_{0} \partial_{X}^{3} A+V_{0} \partial_{X} A=\mathcal{C}_{X}[A] .
$$

The extra term $\mathcal{C}$ is a smooth functional of $A$, and is made necessary at a certain order of the asymptotic expansion by matching the inner flow on the dividing streamlines.

The plan of the paper is as follows. In section 2, we formulate the problem and give the main assumptions used in our study. Section 3 displays the equations of the outer flow and gives the local solutions around the singularity. The critical-layer flow is examined in section 4, where, in particular, the description of this flow is refined in section 4.5 by a better parametrization of the streamlines. The outcome is an amplitude evolution equation of the Korteweg-de Vries type, but with a novel nonlinear term. The flow within the defining separatrices within the critical layer (the so-called "cat's eye" flow) is analyzed using an extension of the Prandtl-Batchelor theorem (Batchelor (1956), see also Rhines and Young (1982)) to slowly-evolving solitary-wave motions, described in Appendix B. In section 5, we show that slow diffusion of the critical layer has a negligible effect on the amplitude equation. The evolution equation is examined in section 6 , and a solitary-wave solution is found whose characteristics depend on the outer flow. In section 7 , we demonstrate the existence of an infinity of modes which are able to generate a solitary wave for different classical mean velocity profiles. The case of a constant shear flow for the mean velocity profile is studied in section 8 which enables us to find an analytical expression for the outer flow, and so we can explicitly determine the integration constants at each order. Section 9 offers some concluding remarks.

\section{Formulation}

We consider a steady and horizontal parallel shear flow $U(y)$ in a Cartesian frame $(x, y)$ centred at a latitude whose directions are east for $x$ and west for $y$. We suppose that the fluid is confined between two rigid walls $y_{1}, y_{2}$ either or both of which may be at infinity. We focus on two-dimensional neutral wave disturbances having a real phase speed $c$. In a frame of reference moving with the wave $x^{\prime}=x-c t$, we may write the total streamfunction $\Psi$ as

$$
\Psi=\int(U(y)-c) d y+\epsilon \psi
$$

where $\epsilon$ is a measure of the disturbance amplitude and is taken to be very small, while $\psi$ is the perturbative streamfunction. The dimensionless equation of motion is the vorticity equation in the $\beta$-plane

$$
\left\{\partial_{t}+(U-c) \partial_{x^{\prime}}\right\} \Delta \psi+\epsilon J(\Delta \psi, \psi)+\left(\beta-U^{\prime \prime}\right) \partial_{x^{\prime}} \psi=\frac{1}{R} \Delta^{2} \psi
$$

$R$ is the Reynolds number and is assumed to be very large; indeed, the critical layer scaling will impose $1 / R=\lambda \epsilon^{\frac{7}{4}}$ where $\lambda$ is an $O(1)$ constant. A body force balances the diffusion of the mean flow $U$ and will appear in the equation of the inner flow. The equation (3) is supplemented by the boundary conditions at the rigid walls,

$$
\psi=0 \quad \text { at } \quad y=y_{1}, y=y_{2} .
$$


The continuity of the leading-order vorticity inside the critical layer, without using thin viscous boundary layers along the separatrices is made possible due to small $O\left(\epsilon^{\frac{1}{2}}\right)$ jumps in the mean vorticity. This conjecture by Habeman (Haberman (1972)) was demonstrated by Brown and Stewartson (1978) in a study of an unsteady, nonlinear and viscous Rossby wave critical layer; the latter acts as a source of vorticity. Here we focus on the long-time asymptotic régime after the critical-layer formation stage characterized by an $O\left(\epsilon^{\frac{1}{2}}\right)$ vorticity spreading throughout the domain by diffusive action. This outward diffusion from the critical layer generates a distorted mean flow. The shear velocity is therefore decomposed in the form

$$
U(y)=U_{0}(y)+\epsilon^{\frac{1}{2}} U_{1}(y)+\epsilon U_{2}(y)+\ldots
$$

$U_{0}(y)$ is the initial velocity profile, $U_{1}(y), U_{2}(y) \ldots$ are the outcome of its interaction with the Rossby wave. The initial wave speed $c_{0}$ is slightly modified by the interaction

$$
\text { and } c=c_{0}+\epsilon^{\frac{1}{2}} c_{1}+\epsilon c_{2}+\ldots
$$

The steady linear inviscid disturbance equation is then,

$$
\left(U_{0}(y)-c_{0}\right) \Delta \psi^{(0)}+\left(\beta-U_{0}^{\prime \prime}(y)\right) \psi^{(0)}=0 .
$$

This equation is singular at $y=y_{c}$ where the phase velocity of the wave perturbation equals the given flow velocity. Hence, this equation fails in the critical layer where $y \simeq y_{c}$. Then transience, nonlinearity or viscosity must be reintroduced in the critical-layer leading-order solution. Here, nonlinearity, together with slow transience, is chosen as being more appropriate for the high Reynolds number flows of geophysical motions. Nevertheless, viscosity is later introduced to render the inviscid solution unique. An inner asymptotic expansion with scalings valid in the critical layer for a long-time régime is considered in conjunction with the outer expansion. By the method of matched asymptotic expansions, these are matched at the edges of the critical layer. In order to obtain a balance between the effects of quadratic nonlinearity and dispersion, we introduce the scaled variables,

$$
X=\epsilon^{\frac{1}{4}} x^{\prime}, \quad T=\epsilon^{\frac{5}{4}} t .
$$

Note that the usual scaling for a KdV equation is expected to be (see (Redekopp (1977)) for a discussion in the present context),

$$
X=\epsilon^{\frac{1}{2}} x^{\prime}, \quad T=\epsilon^{\frac{3}{2}} t .
$$

However, it turns out that this traditional KdV scaling is not valid here, because a matching between the flows in the critical layer characterized by unbounded or bounded streamlines respectively cannot then be achieved. The nonlinear terms automatically appear in the inner flow at the leading order since we aim to remove the apparent critical-layer singularities with nonlinear terms. As a result, our scaling (8) is determined by the need to introduce the dispersive term in $\partial_{X}^{3} A$ into the critical-layer flow at the right order; the analysis will show that dispersion must appear at the order $\epsilon^{2}$ in the velocity field in order to balance the functional term in (1) for $\partial_{T} A$. With (9), the dispersive term will appear too late in the outer flow. Note that our present scaling implies that the dispersive effects are stronger, and the time scale is shorter, than that for the usual KdV dynamics.

The perturbation streamfunction is then expanded as

$$
\psi=\psi^{(0)}+\epsilon^{\frac{1}{2}} \psi^{(1)}+\epsilon \psi^{(2)}+\epsilon^{\frac{3}{2}} \ln \epsilon \psi^{(3)}+\epsilon^{\frac{3}{2}} \psi^{(4)} \ldots
$$

The need for the presence of a logarithmic dependence in $\epsilon$ will appear later on.

\section{Outer flow}

\subsection{The singular mode}

Substitution of (10) into (3) yields, at the leading order the equation determining the singular neutral mode. Thus we set out

$$
\psi^{(0)}=A^{*}(X, T) \phi(y),
$$

where $A^{*}$ is the mode amplitude and $\phi(y)$ then satisfies the Rayleigh-Kuo equation

$$
\mathcal{L}_{0}(\phi)=\partial_{y}^{2} \phi+\frac{\beta-U_{0}^{\prime \prime}(y)}{U_{0}(y)-c_{0}} \phi=0 .
$$


We assume that there is a critical layer at $y=y_{c}$ where $U_{0}\left(y_{c}\right)=c_{0}$ and $U_{0}^{\prime}\left(y_{c}\right) \neq 0$. We can therefore redefine the amplitude by $A^{*}=U_{0}^{\prime} A$. Then the local Frobenius solutions of (12) with $\eta=y-y_{c}$, are

$$
\begin{aligned}
\phi_{a} & =\eta+\sum_{n=2}^{\infty} a_{0, n} \eta^{n}, \\
\phi_{b} & =1+\sum_{n=2}^{\infty} b_{0, n} \eta^{n}+b_{0} \phi_{a}(\eta) \ln \left|\eta^{*}\right|,
\end{aligned}
$$

with the first coefficients given by (the mean flow is evaluated at the critical level):

$$
\begin{gathered}
a_{0,2}=\frac{1}{2} b_{0}, \quad a_{0,3}=\frac{1}{6}\left(\bar{U}_{0}^{\prime \prime \prime}-\frac{1}{2} b_{0} \bar{\beta}\right), \\
b_{0}=-\frac{\beta-U_{0}^{\prime \prime}}{U_{0}^{\prime}}, \quad b_{0,2}=\frac{1}{2}\left(\bar{U}_{0}^{\prime \prime \prime}-\frac{1}{2} \bar{U}_{0}^{\prime \prime} b_{0}-\frac{3}{2} b_{0}^{2}\right) .
\end{gathered}
$$

The next coefficients are given in (A1). We denote $\bar{b}_{0}=b_{0} / U_{0}^{\prime}, \bar{\beta}=\beta / U_{0}^{\prime}$, while $\eta^{*}$ is a normalized cross-stream coordinate given by $\eta^{*}=\eta / \eta_{0}$ where $\eta_{0}$ will be determined when matching the outer flow with the critical-layer induced flow. We will also use a dimensionless $\beta$-coefficient evaluating the ratio $\beta$ to $U_{0}^{\prime \prime}: \hat{\beta}=\bar{\beta} / b_{0}, \hat{\beta} \rightarrow-1$ if $\beta \gg U_{0}^{\prime \prime}$ and $\hat{\beta} \rightarrow 0$ if $U_{0}^{\prime \prime} \gg \beta$.

On either side of the critical level, the singular mode takes the form,

$$
\begin{aligned}
& \phi^{+}(y)=a^{+} b_{0} \phi_{a}+\phi_{b}, \quad y>y_{c}, \\
& \phi^{-}(y)=a^{-} b_{0} \phi_{a}+\phi_{b}, \quad y<y_{c} .
\end{aligned}
$$

Here, we have already invoked the condition that $\phi$ should be continuous at $y=y_{c}$, a result which will be confirmed later by matching across the critical layer. Next, we impose the boundary conditions (4) at $y_{1,2}$ which at this order imply that

$$
\phi^{+}\left(y_{2}\right)=0, \quad \phi^{-}\left(y_{1}\right)=0 .
$$

These conditions then determine each of $a^{+}$and $a^{-}$in terms of the single remaining parameter, namely $y_{c}$. Eventually, matching across the critical layer will determine the phase jump $[a]_{-}^{+}=a^{+}-a^{-}$; this then completes the determination of $\phi^{+}(y), \phi^{-}(y)$ and we can simply refer to the singular mode as $\phi(y)$. Indeed, we will find that $[a]_{-}^{+}=0$.

\section{2 $O\left(\epsilon^{\frac{3}{2}}\right)$ flow}

Proceeding to the next order, the motion is altered by advection (denoted by the subscript $l$ ) due to the additional flow $U_{1}$, and by the dispersion (denoted by $d$ ). Thus, $\psi^{(1)}$ satisfies the equation

$$
\begin{gathered}
\mathcal{L}_{0}\left(\psi^{(1)}\right)=-\mathcal{L}_{1}(\phi) A^{*}-\phi \partial_{X}^{2} A^{*} \\
\text { where } \quad \mathcal{L}_{i}(\phi)=\frac{U_{i}(y)-c_{i}}{U_{0}(y)-c_{0}} \partial_{y}^{2} \phi-\frac{U_{i}^{\prime \prime}(y)}{U_{0}(y)-c_{0}} \phi, \quad c_{i}=U_{i}\left(y_{c}\right), \quad i=1,2 \ldots
\end{gathered}
$$

The general solution for $\psi^{(1)}$, written in a form displaying the behaviour near the critical layer is

$$
\begin{aligned}
& \psi^{(1)}=\phi_{l}^{(1)} A^{*}(X, T)+\phi_{d}^{(1)} \partial_{X}^{2} A^{*}(X, T)+\phi B^{*}(X, T)= \\
& \left(\sum_{n=0}^{\infty}\left[b_{l, 1, n} \eta^{n} \ln \left|\eta^{*}\right|+c_{l, 1, n} \eta^{n}\right]+\alpha_{l, 1} \phi_{a}+\beta_{l, 1} \phi_{b}\right) A^{*}(X, T) \\
& \quad+\left(\sum_{n=2}^{\infty}\left[c_{d, 1, n} \eta^{n}\right]+\alpha_{d, 1} \phi_{a}+\beta_{d, 1} \phi_{b}\right) \partial_{X}^{2} A^{*}(X, T)+\phi B^{*}(X, T) .
\end{aligned}
$$

Here $B^{*}(X, T)$ is the second-order amplitude of the mode which, like $A(X, T)$ obeys an amplitude equation derived at the next order vis-à-vis the amplitude equation for $A(X, T)$. The explicit values of the 
coefficients $b_{l, 1, n}, c_{l, 1, n} \ldots$ are given to the first order in the Appendix A1. The coefficients $\alpha$ (respectively $\beta$ ) are related to the regular (respectively singular) Frobenius solutions (13) of the homogeneous equation (12). They may take different values above and below the critical layer, indicated where necessary by the superscripts \pm .

We note here that the solutions $\phi_{l, d}^{(1)}$ in (18) can be written in the alternative form

$$
\begin{aligned}
\phi_{l, d}^{(1)} & =\gamma_{l, d} \phi+\phi_{I} \int_{y_{B}}^{y} F \phi_{I I} d y-\phi_{I I} \int_{y_{B}}^{y} F \phi_{I} d y, \\
\text { where } F & =-\frac{\mathcal{L}_{1}(\phi)}{W},-\frac{\phi}{W} \text { for } \phi_{l, d}^{(1)} \text { respectively. }
\end{aligned}
$$

Here $\phi_{I, I I}$ are the two linearly independent solutions to the homogeneous equation and $W$ is the related Wronskian. $\phi_{I, I I}$ can be found explicitly in some cases (see section 8 for the case of a constant shear flow). Also, $y_{B}=y_{1,2}$ for the solutions below and above the critical layer, and the corresponding solutions are then indexed by $\mp$ respectively. This is the procedure followed by Redekopp (1977). However, we have preferred to obtain the solution in the form (18) instead as it allows an explicit analysis of the critical layer. In the form (19), the solutions satisfy the outer boundary condition automatically, while the connection with the solutions in (18) can be found in principle by expanding the integral terms near the critical level. This process would eventually yield linear relationships between the coefficients $\gamma_{l, d}$ in (19) and the coefficients $\beta_{l, d}$ and $\alpha_{l, d}$ in (18). The boundary conditions (4) at $y_{1,2}$ respectively affect these relations. The jumps of the singular Frobenius series coefficients $\beta_{l, d}$ will be calculated by matching across the critical layer. We then deduce the jumps between each $\gamma^{ \pm}$and $\alpha^{ \pm}$via the preceding relations.

We have chosen a more elegant way to obtain the $\alpha^{ \pm}$jumps by applying the Fredholm alternative to the inhomogeneous equation (17); for instance for the component $\phi_{l}^{(1)}$ of the $O\left(\epsilon^{\frac{3}{2}}\right)$ streamfunction the alternative gives

$$
\mathcal{P} \int_{y_{c}}^{y_{B}}\left(\frac{U_{1}^{\prime \prime}(y)}{U_{0}(y)-c_{0}}+\left[\beta-U_{0}^{\prime \prime}(y)\right] \frac{U_{1}(y)-c_{1}}{\left[U_{0}(y)-c_{0}\right]^{2}}\right) \phi^{2} d y=\mathcal{P}\left\{\phi_{l}^{(1)}\left(0^{s}\right) \phi^{\prime}\left(0^{s}\right)-\phi_{l}^{(1)^{\prime}}\left(0^{s}\right) \phi\left(0^{s}\right)\right\},
$$

where $\mathcal{P}$ denotes the regular Cauchy part, that is here

$$
\mathcal{P}\left\{\phi_{l}^{(1)}\left(0^{s}\right) \phi^{\prime}\left(0^{s}\right)\right\}=(1+a) b_{0}\left(c_{l, 1,0}+\beta_{l, 1}\right), \mathcal{P}\left\{\phi_{l}^{(1)^{\prime}}\left(0^{s}\right) \phi\left(0^{s}\right)\right\}=b_{0}\left(a b_{l, 1,0}+\beta_{l, 1}\right)+b_{l, 1,1}+c_{l, 1,1}+\alpha_{l, 1},
$$

while $s=1(-1)$ if $y_{c}<(>) y_{B}$. A similar relation is obtained for the dispersive component $\phi_{d}^{(1)}$.

\section{3 $O\left(\epsilon^{2}\right)$ flow}

The particular solution components become more singular near the critical level than the homogeneous solutions $\phi_{a, b}$ (see (13) at the higher orders. Thus, $\psi^{(2)}$ contains contributions due to the temporal derivative of the mode amplitude $A^{*}$, the quadratic nonlinearity (denoted by the subscript $n$ ) and the dispersive terms in $\partial_{X}^{2}$ and $\partial_{X}^{4}$ (denoted by the subscript $f$ for fourth derivative in $X$ ) with a linear part in $A^{*}$ corresponding to the effect of the additional mean flow on $\Psi^{(0)}$ and $\Psi^{(1)}$. Each contribution will affect the coefficients of the amplitude equation governing the evolution of $A$ :

$$
\begin{gathered}
\mathcal{L}_{0}\left(\psi^{(2)}\right)=\frac{\left[\beta-U_{0}^{\prime \prime}(y)\right] \phi}{\left[U_{0}(y)-c_{0}\right]^{2}} \partial_{T X^{-1}} A^{*}-\left(\frac{\beta-U_{0}^{\prime \prime}(y)}{U_{0}(y)-c_{0}}\right)^{\prime} \frac{\phi^{2}}{U_{0}(y)-c_{0}} \frac{1}{2} A^{* 2}-\left[\mathcal{L}_{1}\left(\phi_{l}^{(1)}\right)+\mathcal{L}_{2}(\phi)\right] A^{*} \\
-\left[\frac{U_{1}(y)-c_{1}}{U_{0}(y)-c_{0}} \phi+\phi_{l}^{(1)}+\mathcal{L}_{1}\left(\phi_{d}^{(1)}\right)\right] \partial_{X}^{2} A^{*}-\phi_{d}^{(1)} \partial_{X}^{4} A^{*}-\mathcal{L}_{1}(\phi) B^{*}-\phi \partial_{X}^{2} B^{*}+\lambda \frac{\partial_{y}^{3} U_{1}(y) X}{U_{0}(y)-c_{0}} .
\end{gathered}
$$


Here, we now use the KdV equation (1) to remove the time-derivative term. The general solution for $\psi^{(2)}$, again expressed in a form appropriate at the neighbourhood of the critical level $y_{c}$ is then given by

$$
\begin{gathered}
\psi^{(2)}=\left\{\sum_{n=0}^{\infty}\left[b_{l, 2, n} \eta^{n} \ln \left|\eta^{*}\right|+c_{l, 2, n} \eta^{n}\right]+\alpha_{l, 2} \phi_{a}+\beta_{l, 2} \phi_{b}\right\} A^{*}+\left\{\sum_{n=0}^{\infty}\left[b_{d, 2, n} \eta^{n} \ln \left|\eta^{*}\right|+c_{d, 2, n} \eta^{n}\right]+\alpha_{d, 2} \phi_{a}+\beta_{d, 2} \phi_{b}\right\} \partial_{X}^{2} A^{*} \\
+\frac{1}{2}\left\{\sum_{n=0}^{\infty}\left[a_{n, 2, n} \eta^{n} \ln ^{2}\left|\eta^{*}\right|+b_{n, 2, n} \eta^{n} \ln \left|\eta^{*}\right|+c_{n, 2, n} \eta^{n-1}\right]+\alpha_{n, 2} \phi_{a}+\beta_{n, 2} \phi_{b}\right\} A^{* 2} \\
+\left\{\sum_{n=0}^{\infty}\left[c_{f, 2, n} \eta^{n}\right]+\alpha_{f, 2} \phi_{a}+\beta_{f, 2} \phi_{b}\right\} \partial_{X}^{4} A^{*}+U_{0}^{\prime}\left\{\sum_{n=0}^{\infty}\left[b_{c, 2, n} \eta^{n} \ln \left|\eta^{*}\right|+c_{c, 2, n} \eta^{n}\right]+\alpha_{c, 2} \phi_{a}+\beta_{c, 2} \phi_{b}\right\} \mathcal{C}[A] \\
+\lambda\left\{\sum_{n=0}^{\infty} b_{v, 2, n} \eta^{n} \ln \left|\eta^{*}\right|+c_{v, 2, n} \eta^{n}+\alpha_{v, 2} \phi_{a}+\beta_{v, 2} \phi_{b}\right\} X+\ldots
\end{gathered}
$$

The explicit values of the coefficients $b_{l, 2, n}, c_{l, 2, n} \ldots$ are given to the first order in the Appendix A1.3. Note that they contain a linear dependence on the coefficients $R_{0}, S_{0}, V_{0}$ of the KdV equation (1), and these coefficients are yet to be determined.

The fourth contribution (denoted by the subscript $c$ ) is caused by the motion inside the critical layer. The presence of a higher-order dispersive term leads to the possible existence in the left-hand side of (1) of a term $D_{0} \partial_{X}^{5} A$, but we will show later that in fact $D_{0}=0$. The coefficients $\alpha$ (respectively $\beta$ ) are again related to the regular (respectively singular) Frobenius solutions (13) of the homogeneous equation (12), and again may take different values above and below the critical layer, indicated where necessary by the superscripts \pm . As above, imposition of the boundary conditions (4) at $y_{1,2}$ yields relations between each $\alpha$ and $\beta$. Also, as above in Section 3.2, the solutions can be expressed in a second form analogous to (19), but it is not necessary to give the details here. The Fredholm alternative gives here

$$
\mathcal{P} \int_{y_{c}}^{y_{B}} \mathcal{L}_{0}\left(\psi^{(2)}\right) \phi d y=\mathcal{P} \int_{y_{c}}^{y_{B}} N H(y) \phi(y) d y=\mathcal{P}\left\{\psi^{(2)}\left(0^{s}\right) \phi^{\prime}\left(0^{s}\right)-\psi^{(2)^{\prime}}\left(0^{s}\right) \phi\left(0^{s}\right)\right\} .
$$

$N H(y)$ is the r.h.s of the equation (21). The last term in (23) can be easily expressed via the previous Frobenius expansion series (22).

\section{Nonlinear critical layer flow}

A balance between the mean flow and the perturbation occurs in the critical layer, which leads to the scaling

$$
\eta=y-y_{c}=\epsilon^{\frac{1}{2}} Y .
$$

The governing vorticity equation (3) is then

$$
\left\{\epsilon^{\frac{3}{2}} \partial_{T}+\left(\Psi_{Y} \partial_{X}-\Psi_{X} \partial_{Y}\right)\right\}\left(\partial_{Y}^{2}+\epsilon^{\frac{3}{2}} \partial_{X}^{2}\right) \Psi+\epsilon^{\frac{3}{2}} \beta \Psi_{X}=\epsilon \lambda\left(\partial_{Y}^{4} \Psi+2 \epsilon^{\frac{3}{2}} \partial_{X}^{2} \partial_{Y}^{2} \Psi+\epsilon^{3} \partial_{X}^{4} \Psi\right)+\epsilon^{3} \lambda F .
$$

Here $F$ is the (viscous) body force, $F=-U_{0}^{\prime \prime \prime}(y)=-\left[U_{0}^{\prime \prime \prime}+\epsilon^{\frac{1}{2}} U_{0}^{I V} Y+\ldots\right]$. The expansion of the outer expansion in terms of $Y$ (Appendix A2) determines the way in which the inner expansion must proceed

$$
\Psi=\epsilon\left\{\Psi^{(0)}+\epsilon^{\frac{1}{2}} \ln \epsilon \Psi^{(1)}+\epsilon^{\frac{1}{2}} \Psi^{(2)}+\epsilon \ln ^{2} \epsilon \Psi^{(3)}+\epsilon \ln \epsilon \Psi^{(4)}+\epsilon \Psi^{(5)}+\ldots\right\} .
$$

We shall describe explicitly the analytical expressions of the critical-layer flow at each order.

\section{1 $O(\epsilon)$}

The leading-order equation is

$$
\left(\Psi_{Y}^{(0)} \partial_{X}-\Psi_{X}^{(0)} \partial_{Y}\right) \Psi_{Y Y}^{(0)}=\lambda \partial_{Y}^{4} \Psi^{(0)}
$$

We take the inviscid limit $\lambda \rightarrow 0$, and hence search for a solution in the form:

$$
\Psi^{(0)}=\Psi_{i}^{(0)}+\lambda \Psi_{v}^{(0)}+O\left(\lambda^{2}\right) .
$$


It is useful to transform the variables into streamfunction coordinates,

$$
(X, Y, T) \rightarrow\left(\xi=X, S=\bar{\Psi}^{(0)}, T\right) .
$$

Note that here, and elsewhere, an overbar denotes division by $U_{0}^{\prime}$, so that, for instance, $U_{0}^{\prime} S=U_{0}^{\prime} \bar{\Psi}^{(0)}=$ $\Psi^{(0)}$. Then, the leading-order vorticity equation reduces to

$$
\begin{gathered}
\Psi_{i, Y}^{(0)} \Psi_{i, Y Y \xi}^{(0)}=0, \\
\text { so that } \Psi_{i, Y Y}^{(0)}=\mathcal{F}^{(0)}(S, T) .
\end{gathered}
$$

Here $\mathcal{F}^{(0)}$ is an arbitrary function at this stage. Next, we get for the viscous term

$$
\begin{gathered}
\Psi_{i, Y}^{(0)} \Psi_{v, Y Y \xi}^{(0)}=\partial_{Y}^{4} \Psi_{i}^{(0)}, \\
\text { or } \quad \Psi_{v, Y Y \xi}^{(0)}=\left\{S_{Y} \mathcal{F}_{S}^{(0)}\right\}_{S} .
\end{gathered}
$$

We require that this viscous correction has the the same basic periodicity as that in the outer flow. For a wave train with a wavelength $2 \pi / \Delta$, we see that after averaging the expression above over one period, and integrating once we get

$$
\mathcal{F}_{S}^{(0)}=\frac{M^{(0)}(T)}{\frac{\Delta}{2 \pi} \int_{-\pi / \Delta}^{\pi / \Delta} S_{Y} d \xi} .
$$

But then, on matching with the outer expansion, we readily find that

$$
M^{(0)}=0, \quad \mathcal{F}^{(0)}=U_{0}^{\prime} .
$$

Hence, we have established that

$$
U_{0}^{\prime} S=\Psi_{i}^{(0)}=U_{0}^{\prime}\left[\frac{1}{2} Y^{2}+A(X, T)\right]
$$

This result agrees, of course, with the classical result of Benney and Bergeron (1969). It demonstrates, inter alia, the continuity of the singular mode through the critical layer, a results which we have already anticipated.

Although the form of the streamlines in the critical layer now depends on the precise form of the amplitude $A(X, T)$, it is useful here to suppose that $A$ has a solitary-wave shape; that is, it decays rapidly as $X \rightarrow \pm \infty$, and otherwise consists of a single hump, with a maximum (minimum) of $A_{0}=A(0)>(<) 0$ at $X=0$. In effect, we are taking the limit $\Delta \rightarrow 0$ in the above. The critical layer is then characterized by a zone in which streamlines overturn, and are enclosed within a shape commonly called the cat's eye (cf. figure 1). These streamlines are separated from the remaining part of the flow by separatrices, on which $S=S_{c}$, whose shape depends on the sign of $A_{0}$. The cat's eye has a classical profile, but now has an infinite period with the following characteristics:

$$
\begin{gathered}
A>0, S_{c}=A_{0}, \text { a centre at } A=0, S=0, \text { a hyperbolic point at } A=A_{0}, S=A_{0}, \\
A<0, S_{c}=0, \text { a centre at } A=A_{0}, S=A_{0}, \text { a hyperbolic point at } A=0, S=0 .
\end{gathered}
$$

Following the nomenclature of Redekopp (1977), the first case is called a depression wave, and the second is an elevation wave; the respective possible solitary waves are then $\mathrm{D}$-waves or E-waves.

The same technique applied at $O\left(\epsilon^{\frac{3}{2}} \ln \epsilon\right)$ gives

$$
\Psi_{i}^{(1)}=\frac{1}{2} b_{0} U_{0}^{\prime} Y A .
$$



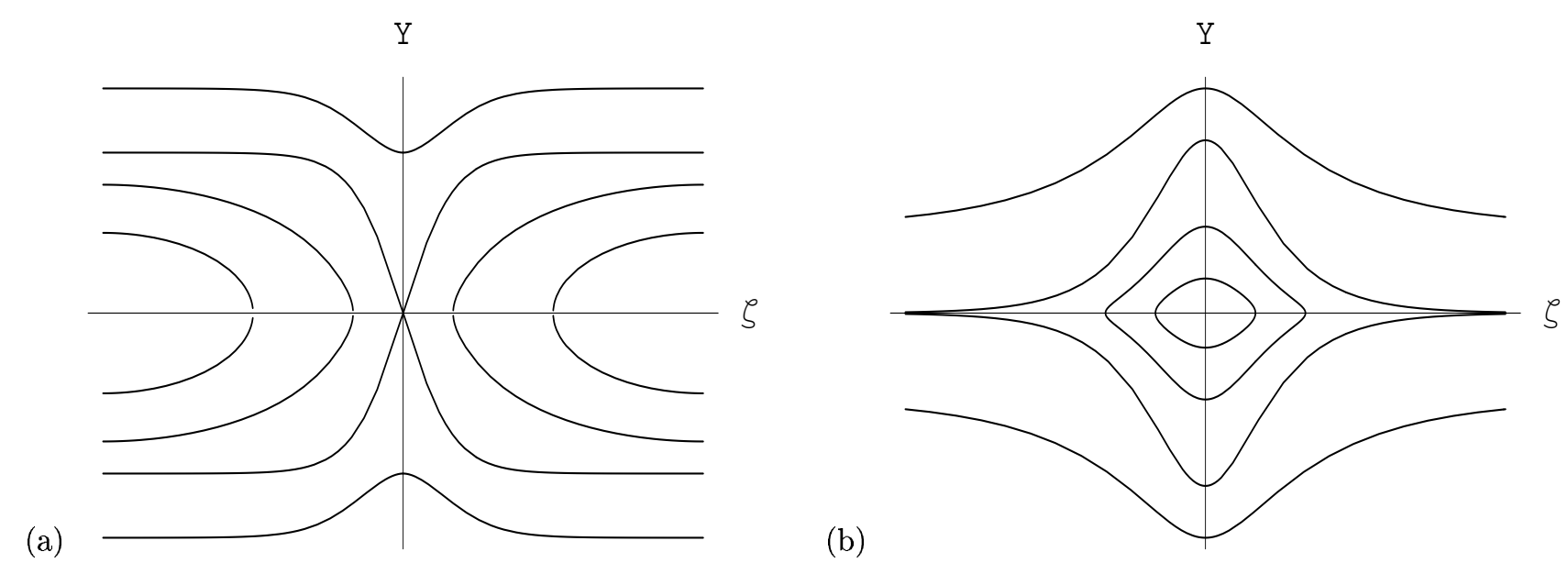

Figure 1: Shape of the nonlinear wave according to the signe of $A$ ( $\zeta$ streamwise coordinate in the frame moving with the nonlinear wave speed, $\zeta=X-V T$ ). (a) D-wave: $A>0$, b) E-wave: $A<0$.

\section{$4.2 O\left(\epsilon^{\frac{3}{2}}\right)$}

\subsubsection{Motion outside the separatrices}

We consider first the motion outside the separatrices, where all streamlines pass to infinity.

Let us denote the total potential vorticity by

$$
Q_{\text {total }}=\Delta \hat{\psi}-f_{0}-\beta y .
$$

Strictly, of course, the usual potential vorticity is $-Q_{\text {total }}$ but we shall retain the present sign convention here. In the critical layer this becomes

$$
Q_{\text {total }}=\frac{1}{\epsilon} \partial_{Y}^{2} \Psi+\epsilon^{\frac{1}{2}} \partial_{X}^{2} \Psi-\epsilon^{\frac{1}{2}} \beta Y-f_{0} .
$$

Thus, the leading-order term is $Q_{0}=U_{0}^{\prime}-f_{0}$. Next, the equation for $Q^{(2)}=\Psi_{Y Y}^{(2)}-\beta Y$ is

$$
\left(S_{Y} \partial_{X}-S_{X} \partial_{Y}\right) Q^{(2)}=\lambda Q_{Y Y}^{(2)} .
$$

The inviscid potential vorticity is then given by

$$
Q_{i}^{(2)}=\Psi_{i, Y Y}^{(2)}-\beta Y=Q(S, T) .
$$

The viscous component of the vorticity is found from

$$
\Psi_{v, Y Y \xi}^{(2)}=\left(S_{Y} Q_{S}\right)_{S} .
$$

As before, we can impose the condition that $\left\langle\Psi_{v, Y Y \xi}^{(2)}\right\rangle=0$ so that we get

$$
\begin{gathered}
Q(S, T)=M^{(2)}(T)\left\{\int_{S_{c}}^{S}\left[I(w, T)-(2 w)^{-\frac{1}{2}}\right] d w+(2 S)^{\frac{1}{2}}\right\}+N^{(2)}(T), \\
\text { where } I(S, T)=\frac{2 \pi}{\Delta}\left(\int_{-\pi / \Delta}^{\pi / \Delta}\{2[S-A(\xi, T)]\}^{\frac{1}{2}} d \xi\right)^{-1} .
\end{gathered}
$$

Note that $S>0$ for an open streamline. We now take the limit $\Delta \rightarrow 0$ to get

$$
I(S, T)=(2 S)^{-\frac{1}{2}} .
$$

Finally, matching with the outer expansion gives

$$
Q(S)=s b_{0} U_{0}^{\prime} \sqrt{2 S}+U_{1}^{\prime} s, \quad \text { where } \quad s=\operatorname{sgn}[Y] .
$$


Integrating $Q$ once, we obtain

$$
\begin{gathered}
\psi_{Y}^{(2)}=b_{0} \mathcal{G}(A, S)+\beta S+U_{1}^{\prime s} Y+\mathcal{U}^{(2)}(\xi, T), \\
\text { where } \mathcal{G}(A, S)=U_{0}^{\prime}\left\{A(\xi, T) \ln [\mathcal{T}(A, S)]+[S(S-A)]^{\frac{1}{2}}\right\}, \\
\text { and } \mathcal{T}(A, S)=\left|\frac{S-A}{A_{0}}\right|^{\frac{1}{2}}+\left|\frac{S}{A_{0}}\right|^{\frac{1}{2}} .
\end{gathered}
$$

Here we recall that $S$ is defined by (28). We omit the subscript $i$ for the velocity and the streamfunction when no confusion is possible. The expansion of $\Psi_{Y}^{(2)}$ when $|Y| \rightarrow \infty$ is

$$
\begin{aligned}
\Psi_{Y}^{(2)}= & b_{0} U_{0}^{\prime}\left[\frac{Y^{2}}{2}+A \ln |Y|+\frac{1}{2} A\left(1-\ln \left|A_{0} / 2\right|\right)\right] \\
& +\beta\left(A+\frac{1}{2} Y^{2}\right)+U_{1}^{\prime} Y^{\prime}+\mathcal{U}^{(2)}(\xi, T)+O\left(1 / Y^{2}\right),
\end{aligned}
$$

which must be matched with

$$
U_{0}^{\prime \prime} \frac{Y^{2}}{2}+U_{1}^{\prime} Y+b_{0} U_{0}^{\prime}\left(1+a^{s}+\ln |Y|-\ln \eta_{0}\right) A+O(1 / Y) .
$$

We then have $\eta_{0}=\sqrt{1 / 2\left|A_{0}\right|}$ and

$$
\mathcal{U}^{(2)}(\xi, T)=U_{0}^{\prime}\left(a^{s}+\frac{1}{2}-\hat{\beta}\right) b_{0} A .
$$

Integrating once again, we get

$$
\begin{aligned}
& \Psi^{(2)}=b_{0} \mathcal{H}(A, S)+\frac{1}{3} \beta S_{Y}(2 A+S)+U_{1}^{\prime} S+\mathcal{U}^{(2)} S_{Y}+\Phi^{(2)}(\xi, T), \\
& \text { where } \mathcal{H}(A, S)=U_{0}^{\prime}\left\{A\left(S_{Y} \ln [\mathcal{T}(A, S)]-s \sqrt{2 S}\right)+\frac{1}{3} s S \sqrt{2 S}\right\} .
\end{aligned}
$$

Matching with the outer flow then gives:

$$
\Phi^{(2)}=\left(c_{l, 1,0}^{s}+\beta_{l, 1}^{s}-\bar{U}_{1}^{\prime s}\right) A^{*}(X, T)+\beta_{d, 1}^{s} \partial_{X^{2}} A^{*}(X, T)+B^{*}(X, T) .
$$

\subsubsection{Motion within the separatrices}

Within the region of closed streamlines, we invoke a modified form (see Appendix B) of the PrandtlBatchelor theorem (Batchelor (1956), Rhines and Young (1982)) to determine the interior potential vorticity. At this order, the interior potential vorticity is a constant. Consequently, by matching on the separatrix $S=S_{c}$ with the inner flow described above, we get that

$$
Q\left(S_{c}\right)=Q_{2}=\frac{1}{2}\left(U_{1}^{\prime+}+U_{1}^{\prime-}\right),
$$

where $Q_{2}$ is a constant. The additional mean flow possesses a vorticity jump through $y_{c}$ given by

$$
\left[U_{1}^{\prime}\right]_{-}^{+}=-2 b_{0} U_{0}^{\prime} Y_{\infty}
$$

$Y_{\infty}$ is the cross-stream location of the separatrix when $\xi$ tends to infinity; from the discussion of section 4.1, we see that $Y_{\infty}=\left(2 A_{0}\right)^{\frac{1}{2}}$ for a D-wave, and $Y_{\infty}=0$ for an E-wave. Also [ $]_{-}^{+}$denotes the jump across the critical layer. The velocity inside the separatrices is readily found

$$
\Psi_{Y}^{(2, c)}=Q_{2} S_{Y}+\beta S+\mathcal{U}_{2}(X, T) .
$$

The superscript $c$ denotes the closed motion within the dividing streamlines. We deduce from this the relationship linking the coefficients $a^{s}$

$$
b_{0}[a]_{-}^{+} A(\xi, T)=0
$$

implying that

$$
a^{+}=a^{-} \text {. }
$$


This result establishes the singular neutral mode has a zero phase jump across the critical layer, as expected (Benney and Bergeron (1969); Redekopp (1977)) and completes the determination of this mode. The integration constant is

$$
\mathcal{U}_{2}(X, T)=b_{0} \mathcal{G}\left(A, S_{c}\right)+\frac{1}{2} Y_{s}\left[U_{1}^{\prime}\right]_{-}^{+}+\mathcal{U}^{(2)}
$$

so the streamfunction is finally

$$
\Psi^{(2, c)}=Q_{2} S+\mathcal{U}_{2} S_{Y}+\frac{1}{3} \beta S_{Y}(2 A+S)+\Phi_{2}(X, T) .
$$

The matching of the streamfunction $\Psi^{(2)}$ on the separatrix $S=S_{c}$ leads to an equality, modulo an additional constant,

$$
\begin{gathered}
\Psi^{(2)}+C^{(2)}=\Psi^{(2, c)} \\
\text { so that } \Phi_{2}(X, T)=\frac{1}{2}\left(\Phi^{(2)+}+\Phi^{(2)-}\right)+\frac{1}{2}\left[C^{(2)+}(T)+C^{(2)-}(T)\right] .
\end{gathered}
$$

Allowing $\Phi^{(2)}$ to possess a jump through the critical layer, we obtain the following relationships,

$$
\begin{gathered}
U_{0}^{\prime}\left(\frac{\left[U_{1}^{\prime \prime}\right]_{-}^{+}}{\beta-U_{0}^{\prime \prime}}+\left[\beta_{l, 1}\right]_{-}^{+}\right) A+\left[C^{(2)}\right]_{-}^{+}=2 b_{0} U_{0}^{\prime} Y_{\infty}\left(2 A-\frac{1}{3} S_{c}\right), \\
\text { and }\left[\beta_{d, 1}\right]_{-}^{+}=0 .
\end{gathered}
$$

The $O\left(\epsilon^{2} \ln ^{2} \epsilon\right)$ flow is simply

$$
\Psi^{(3)}=\frac{1}{8} U_{0}^{\prime} b_{0}^{2} A^{2}
$$

\section{$4.3 \quad O\left(\epsilon^{2} \ln \epsilon\right)$}

\subsubsection{Motion outside the separatrices}

The equation for $\Psi_{Y Y}^{(4)}$ is

$$
U_{0}^{\prime}\left(S_{Y} \partial_{X}-S_{X} \partial_{Y}\right) \Psi_{Y Y}^{(4)}=J\left(\Psi^{(1)}, Q\right)-\partial_{T} \Psi_{Y Y}^{(1)}+\lambda \partial_{Y}^{2} \Psi_{Y Y}^{(4)} .
$$

In the inviscid limit, and with the variable change (27) this reduces to

$$
U_{0}^{\prime} S_{Y} \Psi_{i, Y Y, \xi}^{(4)}=S_{Y}\left(\Psi_{\xi}^{(1)} Q_{S}-\Psi_{S}^{(1)} Q_{\xi}\right),
$$

and hence, on using $(29,30)$ we get that

$$
\Psi_{i, Y Y}^{(4)}=\bar{\Psi}^{(1)} Q_{S}+\mathcal{F}^{(4)}(S, T) .
$$

Further, it is readily shown that the viscous part of the streamfunction is $\Psi_{v}^{(4)}=0$. Imposing the periodicity condition as before, now yields that

$$
\Psi_{i, Y Y}^{(4)}=\frac{1}{2} b_{0} S_{Y} A Q_{S}+M^{(4)}(T)(2 S)^{\frac{1}{2}}+N^{(4)}(T) .
$$

$M^{(4)}$ and $N^{(4)}$ are determined from the behaviour as $Y \rightarrow \pm \infty$, and we find that

$$
M^{(4)}(T)=0, \quad N^{(4)}(T)=0 .
$$

Recalling the expression (31) for $Q$, we finally get that

$$
\Psi_{i, Y Y}^{(4)}=\frac{1}{2} s b_{0}^{2} U_{0}^{\prime} \frac{Y}{\sqrt{2 S}} A .
$$

But, we now see that for the E-wave, there is a singularity as the separatrix is approached, since $S \rightarrow$ $S_{c}=0$ in that case. By contrast, the D-wave remains well-defined as for it, $S_{c}=A_{0} \neq 0$. Hence, in this paper, we will henceforth consider only the D-wave, and defer discussion of the E-wave. However, we 
note here that this singularity in the E-wave is in fact only apparent, as it can be removed by a change of the variable $S$ which better models the distortion of the streamlines in the nonlinear critical layer. Integrating once more, and again applying the matching conditions as $Y \rightarrow \pm \infty$, we find that

$$
\begin{gathered}
\Psi_{Y}^{(4)}=\frac{1}{2} b_{0} Q A+\mathcal{U}^{(4)}(\xi, T), \\
\text { where } \mathcal{U}^{(4)}(\xi, T)=\frac{1}{2} b_{0} U_{0}^{\prime}\left[\left(\beta_{l, 1}^{s}-\bar{U}_{1}^{\prime} s\right) A+\beta_{d, 1} \partial_{X}^{2} A+B\right] .
\end{gathered}
$$

Here, we recall that the superscript ' $s$ ' denotes \pm , that is the values above and below the critical layer respectively, and we note that, from (35), there is no jump in the coefficient $\beta_{d, 1}$. Finally, on integrating $\Psi_{Y}^{(4)}$ in a similar manner, we get that

$$
\Psi^{(4)}=\frac{1}{2} b_{0}^{2} A \mathcal{G}(A, S)+\left(\mathcal{U}^{(4)}+\frac{1}{2} b_{0} U_{1}^{\prime} A\right) S_{Y}+\Phi^{(4)}(\xi, T),
$$

where $\Phi^{(4)}(\xi, T)=\frac{1}{2}\left(b_{l, 2,0} A^{*}+b_{d, 2,0} \partial_{X}^{2} A^{*}+b_{f, 2,0} \partial_{X}^{4} A^{*}+b_{c, 2,0} U_{0}^{\prime} \mathcal{C}[A]\right)+\frac{1}{4}\left(b_{n, 2,0}-b_{0} \bar{b}_{0}\right) A^{* 2}$.

\subsubsection{Motion within the separatrices}

The closed streamlines are characterized by $\Psi_{Y Y}^{(4, c)}=Q_{4}$ where $Q_{4}$ is a constant (see Appendix B), But now, we see that it is impossible to match the vorticity continuously across the separatrix because, from the previous subsection, we have from (37) that

$$
\Psi_{i, Y Y}^{(4)}\left(S_{c}\right)=\frac{1}{2} b_{0}^{2} U_{0}^{\prime} \frac{Y_{s}}{Y_{\infty}} A
$$

where here, $Y_{s}$ denotes the value of $|Y|$ on the separatrix, and $Y_{\infty}=\sqrt{2 A_{0}}$ is its value as $\xi \rightarrow \pm \infty$. Note that this expression also holds formally for the E-wave, but in that case $Y_{\infty}=0$ and so the vorticity is infinite on the separatrix.

The streamwise velocity is given by $\Psi_{Y}^{(4, c)}=Q_{4} S_{Y}+\mathcal{U}_{4}$. Matching the velocity across the separatrix with the expression found in the previous subsection, yields

$$
\mathcal{U}_{4}=\frac{1}{4} b_{0} U_{0}^{\prime}\left(\beta_{l, 1}^{+}+\beta_{l, 1}^{-}\right) A+\frac{1}{2} b_{0} U_{0}^{\prime}\left(\beta_{d, 1} \partial_{X}^{2} A+B\right),
$$

together with the condition

$$
Q_{4} Y_{s}=\frac{1}{2} b_{0} U_{0}^{\prime}\left(b_{0} Y_{\infty}+\frac{1}{2}\left[\beta_{l, 1}\right]_{-}^{+}\right) A
$$

which in turn reduces to

$$
Q_{4}=0, \quad\left[\beta_{l, 1}\right]_{-}^{+}=-2 b_{0} Y_{\infty}, \quad\left(\mathcal{U}^{(4)+}=\mathcal{U}^{(4)-}\right) .
$$

Then, on using (34), we find that the vorticity gradient jump for the distorted mean flow is given by

$$
\left[\bar{U}_{1}^{\prime \prime}\right]_{-}^{+}=-6 b_{0}^{2} Y_{\infty}, \quad\left[C^{(2)}\right]_{-}^{+}=-\frac{2}{3} b_{0} U_{0}^{\prime} S_{c} Y_{\infty}
$$

Finally, the matching of the streamfunction $\Psi^{(4, c)}=\mathcal{U}_{4} S_{Y}+\Phi_{4}(\xi, T)$ with the flow found above in the previous subsection yields

$$
\left[\Phi^{(4)}\right]_{-}^{+}=0, \quad \Phi_{4}=\frac{1}{2} b_{0}^{2} U_{0}^{\prime}\left[b_{0} A \ln \mathcal{T}\left(A, S_{c}\right)-\frac{1}{2} Y_{s} Y_{\infty}\right] A+\Phi^{(4)}+C^{(4)} .
$$

The first equality confirms the implicit assumption that the coefficients of the KdV equation (1) are indeed well-defined and have the same value above and below the critical layer, that is,

$$
R_{0}^{+}=R_{0}^{-}, \quad S_{0}^{+}=S_{0}^{-}, \quad V_{0}^{+}=V_{0}^{-}, \quad D_{0}^{+}=D_{0}^{-} .
$$

Here, we recall that $D_{0}$ is the coefficient of a possible term $\partial_{X}^{5} A$, but will later be shown to be zero. Also the first equality shows that $C^{(4)+}=C^{(4)-}$. 


\section{$4.4 O\left(\epsilon^{2}\right)$}

\subsubsection{Motion outside the separatrices}

The general procedure is now clear, so we shall omit all details and only display essential results. The equation for $\Psi^{(5)}$ is

$$
U_{0}^{\prime}\left(S_{Y} \partial_{X}-S_{X} \partial_{Y}\right) \Psi_{Y Y}^{(5)}=J\left(\Psi^{(2)}, Q\right)-\partial_{T} Q+\lambda\left(\partial_{Y}^{4} \Psi^{(5)}-U_{0}^{\prime \prime \prime}\right) .
$$

Using the variables $S$ and $\xi$ as before, the solution for the inviscid part is

$$
\begin{gathered}
\Psi_{i, Y Y}^{(5)}=\bar{Q}_{S}\left(\Psi^{(2)}-\Pi\left[S_{Y}\right]\right)+\mathcal{F}^{(5)}(S, T), \\
\text { where } \quad \Pi\left[S_{Y}\right]=\int_{-\infty}^{\xi} \frac{\partial_{T} A(x, T)}{S_{Y}(S, x, T)} d x .
\end{gathered}
$$

Applying the secularity condition to the viscous part yields

$$
\mathcal{F}^{(5)}=\left(U_{0}^{\prime \prime \prime}-\frac{1}{3} b_{0} U_{0}^{\prime \prime}\right) S+s\left[M^{(5)}(T)-\frac{1}{2} b_{0} U_{1}^{\prime s}\right] \sqrt{2 S}+U_{2}^{\prime}
$$

Note that it is not necessary to introduce a viscous time scale in the critical layer (compare Redekopp (1977)). Next, we consider the matching with the outer flow as $Y \rightarrow \pm \infty$, which yields $M^{(5)}=U_{1}^{\prime \prime}$. Integrating $\Psi_{Y Y}^{(5)}$ and again applying matching conditions as $Y \rightarrow \pm \infty$, we get

$$
\begin{aligned}
\Psi_{Y}^{(5)}=\frac{1}{3}\left(U_{0}^{\prime \prime \prime}-\frac{1}{3} \beta b_{0}\right) S_{Y}(2 A+S)-2 b_{0}^{2} U_{0}^{\prime} S_{Y} A+s b_{0}\left(\frac{2}{3} \bar{\beta} A+\frac{1}{9} \beta S+\mathcal{U}^{(2)}\right) \sqrt{2 S} \\
+U_{1}^{\prime \prime}[S(S-A)]^{\frac{1}{2}}+U_{2}^{\prime} S_{Y}-s \frac{1}{2} b_{0} \int_{\infty}^{S} \frac{\Pi\left[(w-A)^{\frac{1}{2}}\right]}{[2 w(w-A)]^{\frac{1}{2}}} d w \\
+b_{0} U_{0}^{\prime}\left(\beta_{l, 1} A+\beta_{d, 1} \partial_{X}^{2} A+s b_{0} \sqrt{2 S} A+B\right) \ln [\mathcal{T}(A, S)]+\mathcal{U}^{(5)}(\xi, T)
\end{aligned}
$$

where $\quad \mathcal{U}^{(5)}(\xi, T)=\left(\alpha_{l, 1}+b_{0} \beta_{l, 1}-\frac{1}{2} \bar{U}_{1}^{\prime \prime}\right) A^{*}+\left(\alpha_{d, 1}+\beta_{d, 1} b_{0}\right) \partial_{X}^{2} A^{*}+(1+a) b_{0} B^{*}$.

A second integration yields

$$
\begin{aligned}
\Psi^{(5)}=\left(U_{0}^{\prime \prime \prime}\right. & \left.-b_{0} \frac{\beta}{3}\right)\left(2 A+\frac{1}{2} S\right) \frac{S}{3}+\left(U_{2}^{\prime}-2 b_{0}^{2} U_{0}^{\prime} A+\frac{1}{3} s U_{1}^{\prime \prime} \sqrt{2 S}\right) S \\
& +\bar{b}_{0}\left(\frac{2}{3} \bar{\beta} A+\mathcal{U}^{(2)}\right) \mathcal{G}(A, S)+\frac{\bar{b}_{0} \bar{\beta}}{18}\left\{\left(\frac{3}{2} A+S\right)[S(S-A)]^{\frac{1}{2}}+\frac{3}{2} A^{2} \ln [\mathcal{T}(A, S)]\right\} \\
& -\frac{1}{4} b_{0} \int_{\infty}^{S} \frac{d w_{1}}{\left(w_{1}-A\right)^{\frac{1}{2}}} \int_{\infty}^{w_{1}} \frac{\Pi\left[\left(w_{2}-A\right)^{\frac{1}{2}}\right]-w_{2}^{-\frac{1}{2}} A_{T X^{-1}}}{\left[w_{2}\left(w_{2}-A\right)\right]^{\frac{1}{2}}} d w_{2}-\frac{1}{4} b_{0} \mathcal{K}(A, S) A_{T X^{-1}} \\
& \quad+b_{0} U_{0}^{\prime}\left(\beta_{l, 1} A+\beta_{d, 1} \partial_{X}^{2} A+B\right)\left\{S_{Y} \ln [\mathcal{T}(A, S)]-s \sqrt{2 S}\right\} \\
+ & \frac{1}{2} b_{0}^{2} U_{0}^{\prime} A\left\{2[S(S-A)]^{\frac{1}{2}} \ln [\mathcal{T}(A, S)]-S+A \ln [\mathcal{T}(A, S)]^{2}\right\}+\mathcal{U}^{(5)}(\xi, T) S_{Y}+\Phi^{(5)}(\xi, T)
\end{aligned}
$$

where $\quad \Phi^{(5)}=\left(2-\frac{1}{2} a-\frac{5}{6} \frac{\bar{U}_{0}^{\prime \prime \prime}}{b_{0}^{2}}+\frac{55}{144} \hat{\beta}\right) b_{0}^{2} U_{0}^{\prime} A^{2}+\left(\beta_{l, 2}+c_{l, 2,0}-\bar{U}_{2}^{\prime}\right) A^{*}+\frac{1}{2}\left(\beta_{n, 2}+c_{n, 2,1}\right) A^{* 2}$ $+\left(\beta_{d, 2}+c_{d, 2,0}\right) \partial_{X}^{2} A^{*}+\left(\beta_{f, 2}+c_{f, 2,0}\right) \partial_{X}^{4} A^{*}+\left(\beta_{c, 2}+c_{c, 2,0}\right) U_{0}^{\prime} \mathcal{C}[A]+\left(c_{l, 1,0}+\beta_{B, l, 1}\right) B^{*}+\beta_{B, d, 1} \partial_{X}^{2} B^{*}$.

Here, recalling that $A>0$ for a $\mathrm{D}$-wave,

$$
\mathcal{K}(A, S)=\left(\frac{2}{A}\right)^{\frac{1}{2}}\left\{S \arctan \left[\left(\frac{S-A}{A}\right)^{\frac{1}{2}}\right]-[A(S-A)]^{\frac{1}{2}}-\frac{\pi}{2}(S-A)\right\} .
$$




\subsubsection{Motion within the separatrices}

The streamfunction within the closed streamlines is

$$
\Psi^{(5, c)}=\frac{1}{6} U_{0}^{\prime \prime \prime} S(S+4 A)+Q_{5} S+\mathcal{U}_{5}(X, T) S_{Y}+\Phi_{5}(X, T)
$$

where $Q_{5}$ is a constant. Note that at this order the vorticity is not constant (see (B)). Matching of the vorticity is not possible; indeed, if so it would imply an absence of a dispersive term in the amplitude equation. However, the streamwise velocity must be continuous (due to the necessity to have a continuous pressure via Bernoulli relation), which leads to two conditions; the first determines $\mathcal{U}_{5}$

$$
\mathcal{U}_{5}=\frac{1}{2} \underline{U}_{1}^{\prime \prime} Y_{s} Y_{\infty}+\frac{1}{2}\left[U_{2}^{\prime}\right]_{-}^{+} Y_{s}+b_{0} U_{0}^{\prime}\left(\underline{\beta}_{l, 1} A+\beta_{d, 1} \partial_{X}^{2} A+B\right) \ln \mathcal{T}\left(A, S_{c}\right)+\underline{\mathcal{U}}^{(5)}
$$

where $\underline{f}$ is the mean value of $f$ through the critical level, $\underline{f}=1 / 2\left(f^{+}+f^{-}\right)$.

The second condition yields the evolution equation

$$
\begin{aligned}
\frac{2}{9} \beta b_{0}\left[S_{c} Y_{\infty}-Y_{s}\left(2 A+S_{c}\right)\right]-2 b_{0}^{2} U_{0}^{\prime}(2 A+ & \left.3 S_{c}\right) Y_{s}+2 b_{0}\left(\frac{2}{3} \beta A+\mathcal{U}^{(2)}\right) Y_{\infty} \\
& +2\left(\underline{U}_{2}^{\prime}-Q_{5}\right) Y_{s}+\left[\mathcal{U}^{(5)}\right]_{-}^{+}=b_{0} \int_{\infty}^{S_{c}} \frac{\Pi\left[(S-A)^{\frac{1}{2}}\right]}{[2 S(S-A)]^{\frac{1}{2}}} d S .
\end{aligned}
$$

Since this equation must be valid when $A=0$ (or when $S_{c} \rightarrow \infty$ ), we can obtain an expression for $Q_{5}$

$$
Q_{5}=\underline{U}_{2}^{\prime}-3 b_{0}^{2} U_{0}^{\prime} S_{c}, \quad S_{c} \neq 0 .
$$

In order to evaluate the integral on the right-hand side of (45), we can use the assumption that the solution is a wave with constant speed $V$. $\Pi$ is thus straightforwardly computed by substituting $\partial_{T} A=-V \partial_{X} A$. After integrating over $S$, for the $\mathrm{D}$-wave, this gives

$$
2 b_{0} V\left(Y_{\infty}-Y_{s}\right) .
$$

Then, the temporal derivative $A_{T X^{-1}}$ is introduced through $V$ yielding the integrated KdV equation, and by factorizing by $A, A^{2}, \partial_{X}^{2} A \ldots$, we find that the coefficients of the equation (1) are given by

$$
\begin{aligned}
V_{0} & =\left(a-1-\frac{4}{9} \hat{\beta}\right) b_{0} U_{0}^{\prime} S_{c}+\frac{1}{4} \frac{\left[\alpha_{l, 1}\right]_{-}^{+}}{\bar{b}_{0}} Y_{\infty} \\
R_{0} & =4\left(1+\frac{1}{9} \hat{\beta}\right) b_{0} U_{0}^{\prime} \\
S_{0} & =\frac{1}{4}\left[\alpha_{d, 1}\right]_{-}^{+} \frac{Y_{\infty}}{\bar{b}_{0}} \\
D_{0} & =0
\end{aligned}
$$

while the right-hand side of (1) is given by

$$
\mathcal{C}[A]=\frac{1}{2} b_{0} U_{0}^{\prime} Y_{\infty}\left(1-a+\frac{5}{9} \hat{\beta}\right) A Y_{s}-\frac{1}{4} \frac{\left[\alpha_{l, 1}\right]_{-}^{+}}{\bar{b}_{0}} A Y s-\frac{1}{4}\left[\alpha_{d, 1}\right]_{-}^{+} \frac{Y_{s}}{\bar{b}_{0}} \partial_{X}^{2} A
$$

This last term breaks the characteristic KdV invariance with respect to the transformation: $X^{\prime}=$ $\delta X, T^{\prime}=\delta^{3} T, A^{\prime}=\delta^{-2} A$. It also prevents one from extracting a symmetric operator enabling one to analytically study the stability of the possible solutions (Pelinovskiy and Grimshaw (1996)). These expressions are the main results from our critical layer analysis. Note, in particular, that the constants $V_{0}, R_{0}$ and $S_{0}$ are not independent of $A$ because they depend explicitly on $A_{0}=A(X-V T=0)$. They are also dependent on the $O\left(\epsilon^{2}\right)$ velocity jumps: $\left[\alpha_{l, 1}\right]_{-}^{+}$and $\left[\alpha_{d, 1}\right]_{-}^{+}$whose expressions involve both the outer and inner flows are in general given by (see section 8 for a special case).

$$
\left[\alpha_{l, 1}\right]_{-}^{+}=-\mathcal{P} \int_{y_{1}}^{y_{2}}\left(\frac{U_{1}^{\prime \prime}(y)}{U_{0}(y)-c_{0}}+\left[\beta-U_{0}^{\prime \prime}(y)\right] \frac{U_{1}(y)-c_{1}}{\left[U_{0}(y)-c_{0}\right]^{2}}\right) \phi^{2}(y) d y+a b_{0}\left[\beta_{l, 1}\right]_{-}^{+}+(a+1) b_{0}\left[c_{l, 1,0}\right]_{-}^{+}
$$




$$
\left[\alpha_{d, 1}\right]_{-}^{+}=\int_{y_{1}}^{y_{2}} \phi^{2}(y) d y+a b_{0}\left[\beta_{d, 1}\right]_{-}^{+},
$$

where we recall that $\mathcal{P}$ is the Cauchy principal part of the integral. $\left[\beta_{l, 1}\right]_{-}^{+},\left[c_{l, 1,0}\right]_{-}^{+}$and $\left[\beta_{d, 1}\right]_{-}^{+}$have been determined by the inner-flow study.

Continuing, we find that the matching of the streamfunction across the separatrices yields the following jumps

$$
\begin{gathered}
{\left[\beta_{n, 2}\right]_{-}^{+}=0, \quad\left[\beta_{d, 2}\right]_{-}^{+}=-2 \beta_{d, 1} b_{0} Y_{\infty}, \quad\left[\beta_{f, 2}\right]_{-}^{+}=0, \quad\left[\beta_{c, 2}\right]_{-}^{+}=0,} \\
{\left[\beta_{l, 2}\right]_{-}^{+}=2 b_{0} \underline{\beta}_{l, 1} Y_{\infty}-\left[\bar{U}_{2}^{\prime}\right]_{-}^{+}-\left[c_{l, 2,0}\right]_{-}^{+}-2 \bar{U}_{1}^{\prime \prime} Y_{\infty},} \\
{\left[c_{l, 2,0}\right]_{-}^{+}=4 Y_{\infty}\left(\bar{b}_{0} Q_{2}+b_{0} \underline{\beta}_{l, 1}-2 \underline{\underline{U}}_{1}^{\prime \prime}\right)-\frac{\left[\bar{U}_{2}^{\prime \prime}\right]_{-}^{+}}{b_{0}}+\left[\bar{U}_{2}^{\prime}\right]_{-}^{+}, \quad\left[C^{(5)}\right]_{-}^{+}=\left\{\frac{4}{3} \bar{U}_{1}^{\prime \prime} Y_{\infty}+\left[U_{2}^{\prime}\right]_{-}^{+}\right\} S_{c},}
\end{gathered}
$$

together with the determination of the constant of integration

$$
\begin{aligned}
\Phi_{5}=-\frac{1}{4} b_{0} \int_{\infty}^{S_{c}} \frac{d w}{(w-A)^{\frac{1}{2}}} \int_{\infty}^{w} \frac{\Pi\left[(r-A)^{\frac{1}{2}}\right]-r^{-\frac{1}{2}} A_{T X-1}}{[r(r-A)]^{\frac{1}{2}}} d r-\frac{1}{4} b_{0} \mathcal{K}\left(A, S_{c}\right) A_{T X^{-1}} \\
+\frac{1}{2} b_{0}^{2} Y_{s} Y_{\infty}\left\{\frac{3}{2}+a-\ln \left[\mathcal{T}\left(A, S_{c}\right)\right]-\frac{1}{4} \hat{\beta}\right\} A^{*}+\frac{1}{2}\left[\alpha_{l, 1} A^{*}+\alpha_{d, 1} \partial_{X}^{2} A^{*}\right]_{-}^{+} Y_{s} \\
+\left\{1+2 a-\frac{1}{2} \hat{\beta}+\ln [\mathcal{T}(A, S c)]\right\} \frac{1}{2} b_{0}^{2} U_{0}^{\prime} A^{2} \ln \left[\mathcal{T}\left(A, S_{c}\right)\right]+\frac{1}{2}\left(4-a-\frac{5}{3} \frac{\bar{U}_{0}^{\prime \prime \prime}}{b_{0}^{2}}+\frac{55}{72} \hat{\beta}\right) b_{0}^{2} U_{0}^{\prime} A^{2} \\
+\beta b_{0} \frac{S_{c}}{36} Y_{s} Y_{\infty}-\frac{1}{2} b_{0}^{2} S_{c} U_{0}^{\prime}\left(A+4 S_{c}\right)-\frac{\beta}{18} b_{0} \frac{S_{c}}{6}\left(4 A+S_{c}\right)+\underline{\beta}_{l, 2} A^{*} \\
+\frac{1}{2} \beta_{n, 2} A^{* 2}+\left(\underline{\beta}_{d, 2}+\underline{c}_{d, 2,0}\right) \partial_{X}^{2} A^{*}+\beta_{f, 2} \partial_{X}^{4} A^{*}+\beta_{c, 2} U_{0}^{\prime} \mathcal{C}[A] \\
+\underline{C}^{(5)}+\left(\underline{U}_{2}^{\prime}-Q_{5}\right) S_{c}+\left(\underline{c}_{l, 1,0}+\beta_{b, l, 1}\right) B^{*}+\beta_{B, d, 1} \partial_{X}^{2} B^{*} .
\end{aligned}
$$

The jumps in the $\alpha$-coefficients at the second-order are

$$
\begin{gathered}
{\left[\alpha_{n, 2}\right]_{-}^{+}=R_{0} \mathcal{P} \int_{y_{1}}^{y_{2}} \frac{\beta-U_{0}^{\prime \prime}(y)}{\left[U_{0}(y)-c_{0}\right]^{2}} \phi^{2}(y) d y+\mathcal{P} \int_{y_{1}}^{y_{2}}\left(\frac{\beta-U_{0}^{\prime \prime}(y)}{U_{0}(y)-c_{0}}\right)^{\prime} \frac{\phi^{3}(y)}{U_{0}(y)-c_{0}} d y} \\
{\left[\alpha_{d, 2}\right]_{-}^{+}=S_{0} \mathcal{P} \int_{y_{1}}^{y_{2}} \frac{\beta-U_{0}^{\prime \prime}(y)}{\left[U_{0}(y)-c_{0}\right]^{2}} \phi^{2}(y) d y+\int_{y_{1}}^{y_{2}} \phi(y) \phi_{l}^{(1)}(y) d y+\int_{y_{1}}^{y_{2}} \frac{U_{1}(y)-c_{1}}{U_{0}(y)-c_{0}} \phi(y)^{2} d y} \\
-\mathcal{P} \int_{y_{1}}^{y_{2}}\left(U_{1}^{\prime \prime}(y)+\left[\beta-U_{0}^{\prime \prime}(y)\right] \frac{U_{1}(y)-c_{1}}{U_{0}(y)-c_{0}}\right) \frac{\phi(y) \phi_{d}^{(1)}(y)}{U_{0}(y)-c_{0}} d y+2 \beta_{d, 1} b_{0}^{2}(2+a) Y_{\infty}, \\
{\left[\alpha_{l, 2}\right]_{-}^{+}=\mathcal{P} \int_{y_{1}}^{y_{2}}\left(U_{1}^{\prime \prime}(y)+\left[\beta-U_{0}^{\prime \prime}(y)\right] \frac{U_{1}(y)-c_{1}}{U_{0}(y)-c}\right)\left(\frac{U_{1}(y)-c_{1}}{U_{0}(y)-c} \phi(y)-\phi^{(1)}(y)\right) \frac{\phi(y)}{U_{0}(y)-c} d y} \\
+V_{0} \mathcal{P} \int_{y_{1}}^{y_{2}} \frac{\beta-U_{0}^{\prime \prime}(y)}{\left[U_{0}(y)-c_{0}\right]^{2}} \phi^{2}(y) d y-\mathcal{P} \int_{y_{1}}^{y_{2}}\left(\frac{U_{2}^{\prime \prime}(y)}{U_{0}(y)-c_{0}}+\left[\beta-U_{0}^{\prime \prime}(y)\right] \frac{U_{2}(y)-c_{2}}{\left[U_{0}(y)-c_{0}\right]^{2}}\right) \phi^{2}(y) d y \\
{\left[\alpha_{f, 2}\right]_{-}^{+}=-\mathcal{P} \int_{y_{1}}^{y_{2}} \phi_{d}^{(1)} \phi(y) d y} \\
{\left[\alpha_{c, 2}\right]_{-}^{+}=-\mathcal{P} \int_{y_{1}}^{y_{2}} \frac{\beta-U_{0}^{\prime \prime}(y)}{\left[U_{0}(y)-c_{0}\right]^{2}} \phi^{2}(y) d y .}
\end{gathered}
$$

The expansion can be continued, and we have explicitly calculated the terms at $O\left(\epsilon^{5 / 2} \ln ^{2} \epsilon\right), O\left(\epsilon^{5 / 2} \ln \epsilon\right)$ and $O\left(\epsilon^{5 / 2}\right)$. We shall not give the details here, but note that the outcome is the determination of the evolution equation for the second-order amplitude $B$ obtained at the order $\epsilon^{\frac{5}{2}}$. The $O\left(\epsilon^{2}\right) \ln \epsilon$ streamwise velocity reveals to be discontinuous on the separatrices. 


\subsection{Redefinition of the separatrix}

In the previous subsections, we have determined the flow within the critical layer by using the variable $S=\Psi^{(0)}$ which gives a first approximation of the location of the dividing streamlines, namely $S_{c}=A(0)$. But an examination at the next order of the expansion shows that this definition is incomplete. The cat's eye is not symmetric with respect to the critical level and a better variable $\tilde{S}$ is needed in order to correctly describe the geometry of the critical layer,

$$
S=\tilde{S}+\delta \varphi^{(1)}(\tilde{S})+\epsilon^{\frac{1}{2}} \varphi^{(2)}(\tilde{S})+\delta^{2} \varphi^{(3)}(\tilde{S})+\epsilon^{\frac{1}{2}} \delta \varphi^{(4)}(\tilde{S},)+\epsilon \varphi^{(5)}(\tilde{S})+\epsilon^{\frac{1}{2}} \delta^{2} \varphi^{(6)}(\tilde{S})+\epsilon \delta \varphi^{(7)}(\tilde{S}) .
$$

The functions $\varphi^{(i)}$ are determined by ensuring that there is zero velocity at the stagnation point, a property which was not previously satisfied. We also check that the velocity is zero at infinity in the core of the two halves of the cat's eye. The comparison with the preceding section can be made with the use of another cross-stream coordinate $\tilde{Y}$. Indeed, $\tilde{S}$ can be written as a function of $S$ or equivalently and more simply in terms of $\tilde{Y}$ :

$$
\begin{gathered}
\tilde{S}=S+\delta \bar{\Psi}^{(1)}(S)-\epsilon^{\frac{1}{2}} \varphi^{(2)}(S)+\delta^{2} \bar{\Psi}^{(3)}(S)+\ldots=\frac{1}{2} \tilde{Y}^{2}+A, \\
\text { with } \quad \tilde{Y}=Y+\delta \frac{1}{2} b_{0} A+\epsilon^{\frac{1}{2}}\left(\bar{\beta} A+\overline{\tilde{U}}_{2}\right)+\epsilon^{\frac{1}{2}} \delta \overline{\mathcal{U}}^{(4)}+\ldots
\end{gathered}
$$

The separatrix is then defined by

$$
\tilde{Y}_{s}=\left[2\left(\tilde{S}_{c}-A\right)\right]^{\frac{1}{2}} \text { and } \quad \tilde{S}_{c}=A(0) .
$$

The expressions for $\varphi$ are

$$
\begin{gathered}
\varphi^{(1)}(S)=-\bar{\Psi}^{(1)}, \varphi^{(2)}=-\left(\bar{\beta} A+\overline{\tilde{U}}_{2}\right) S_{Y}, \varphi^{(3)}=0 \\
\varphi^{(4)}=\frac{1}{2} \bar{b}_{0}\left(\beta A+\tilde{\mathcal{U}}_{2}\right) A-\overline{\mathcal{U}}^{(4)} S_{Y}, \varphi^{(6)}=\frac{1}{2} \bar{b}_{0} \mathcal{U}^{(4)} A-\left(\overline{\mathcal{U}}^{(6)}+\frac{1}{8} \bar{\beta} b_{0}^{2} A^{2}\right) S_{Y} .
\end{gathered}
$$

We obtain the new expansion:

$$
\tilde{\Psi}=\epsilon U_{0}^{\prime} \tilde{S}+\epsilon^{\frac{3}{2}} \tilde{\Psi}^{(2)}+\epsilon^{2} \ln \epsilon^{2} \tilde{\Psi}^{(3)}+\epsilon^{2} \ln \epsilon \tilde{\Psi}^{(4)}+\epsilon^{2} \tilde{\Psi}^{(5)}+\epsilon^{\frac{5}{2}} \ln ^{2} \epsilon \tilde{\Psi}^{(6)}+\epsilon^{\frac{5}{2}} \ln \epsilon \tilde{\Psi}^{(7)}+\ldots
$$

The integration constants $\mathcal{F}$ are not altered by the change of variables. We give in Appendix $\mathrm{C}$ the new expressions of potential vorticity, velocity and streamfunction to the first orders. We describe below the $O\left(\epsilon^{2}\right)$ term that yields the evolution equation.

With this new variable, the PV at $O\left(\epsilon^{2}\right)$ is now given by (the notation follows that of subsections 4.4.1 and 4.4.2),

$$
\tilde{Q}^{(5)}=\tilde{\Psi}_{Y Y}^{(5)}+\beta\left(\bar{\beta} A+\overline{\tilde{U}}_{2}\right), \quad \tilde{Q}^{(5, c)}=Q_{5}
$$

whereas the vorticity is $\quad \tilde{\Psi}_{Y Y}^{(5)}=\Psi_{Y Y}^{(5)}-\left(\bar{\beta} A+\overline{\tilde{U}}_{2}\right)\left(\beta+Q_{\tilde{S}} S_{Y}\right), \quad \tilde{\Psi}_{Y Y}^{(5, c)}=Q_{5}-\beta\left(\bar{\beta} A+\overline{\tilde{U}}_{2}\right)$.

The definition of $\tilde{\mathcal{U}}_{2}$ is given in the Appendix C. Again matching of the potential vorticity cannot be satisfied, and it is discontinuous through the separatrix. The new velocity is

$$
\begin{gathered}
\tilde{\Psi}_{Y}^{(5)}=\frac{1}{3} b_{0}\left(U_{0}^{\prime \prime \prime}-\frac{1}{3} \beta b_{0}\right)(2 A+\tilde{S})\left(\tilde{S}_{\tilde{Y}}-s \tilde{Y}_{s}\right)-2 b_{0}^{2} U_{0}^{\prime} A\left(\tilde{S}_{\tilde{Y}}-s \tilde{Y}_{s}\right)+s b_{0}\left(\frac{2}{3} \beta A+\mathcal{U}^{(2)}\right)\left(\sqrt{2 \tilde{S}}-Y_{\infty}\right) \\
+s \frac{1}{9} \beta b_{0}\left(\tilde{S} \sqrt{2 \tilde{S}}-\tilde{S}_{c} Y_{\infty}\right)-s \frac{1}{2} b_{0}\left(K[A, \tilde{S}]-K\left[A, \tilde{S}_{c}\right]\right)-\bar{\beta} \tilde{S}_{\tilde{Y}}\left(\beta A+\tilde{\mathcal{U}}_{2}\right) \\
\tilde{\Psi}_{Y}^{(5, c)}=-\bar{\beta} \tilde{S}_{\tilde{Y}}\left(\beta A+\tilde{\mathcal{U}}_{2}\right),
\end{gathered}
$$

and is trivially continuous through the separatrices thanks to the appropriate choice of $\varphi^{(5)}$ and $\varphi^{(5, c)}$

$$
\begin{aligned}
\varphi^{(5)}(S)=\frac{1}{2} \frac{\varphi^{(2) 2}}{S_{Y}^{2}}-\varphi^{(2)} \bar{Q}-s \frac{1}{3}\left(\bar{U}_{0}^{\prime \prime \prime}-\frac{1}{3} \beta \bar{b}_{0}\right)(2 A+S) Y_{s} S_{Y}+2 s b_{0}^{2} Y_{s} A S_{Y}-s \bar{b}_{0}\left(\frac{2}{3} \beta A+\mathcal{U}^{(2)}\right) Y_{\infty} S_{Y} \\
+s \bar{b}_{0}\left(\frac{1}{2} K\left[A, \tilde{S}_{c}\right]-\frac{1}{9} \beta \tilde{S}_{c} Y_{\infty}\right) S_{Y}-\overline{\mathcal{U}}^{(5)} S_{Y}-\bar{U}_{1}^{\prime \prime}[S(S-A)]^{\frac{1}{2}} S_{Y} \\
-2 \bar{U}_{2}^{\prime}(S-A)-b_{0}\left(\beta_{l, 1} A+\beta_{d, 1} \partial_{X}^{2} A+s b_{0} \sqrt{2 S} A+B\right) S_{Y} \ln [\mathcal{T}(A, S)], \quad
\end{aligned}
$$




$$
\varphi^{(5, c)}=\frac{1}{2} \frac{\varphi^{(2) 2}}{S_{Y}^{2}}-\bar{Q}_{5} S_{Y}^{2}-\varphi^{(2)} \bar{Q}_{2}-\overline{\tilde{U}}_{5} S_{Y}
$$

$\varphi^{(5)}=\varphi^{(5, c)}$ on the separatrix implies $(45)$ and the expression of $\tilde{\mathcal{U}}_{5}$

$$
\tilde{\mathcal{U}}_{5}=\underline{\mathcal{U}}^{(5)}+\frac{1}{2}\left(\underline{U}_{1}^{\prime \prime} Y_{\infty}+\left[U_{2}^{\prime}\right]_{-}^{+}\right) Y_{s}+b_{0} U_{0}^{\prime}\left(\underline{\beta}_{l, 1} A+\beta_{d, 1} \partial_{X}^{2} A+B\right) \ln \mathcal{T}\left(A, \tilde{S}_{c}\right) .
$$

The new streamfunction is defined by

$$
\tilde{\Psi}^{(5)}=\Psi^{(5)}+U_{0}^{\prime} \varphi^{(5)}+\varphi^{(2)} \Psi_{\tilde{S}}^{(2)} .
$$

We easily check that $\tilde{\Psi}^{(5)}=\tilde{\Psi}^{(5, c)}$ on the separatrix. The jump relationships are identical to these found with the plain inner expansion. The amplitude equation is not modified by this new separatrix definition.

\section{The distorted mean flow}

We recall that the matching of the open- and closed-streamline flows within the nonlinear critical layer involved a distorted mean flow $U_{1}, U_{2} \ldots$. The latter is created as the critical layer is forming in a transition stage, where the initial unsteady process is caused by vorticity diffusion from the critical layer. The mean flow gradually spreads out from the critical layer in two regions on either side, called diffusion boundary layers, and the distortion diminishes with the distance from the critical layer. The critical layer evolves in a complicated way at the beginning and then in a decelerated mode (Fritts (1978)). For large $T=\epsilon^{\frac{5}{4}} t$ in a stable flow, in the frame moving with the nonlinear-wave speed, a quasi-steady régime is established, and the time evolution is given by a slow diffusion process. The diffusion length $\sqrt{\lambda T}$ increases with $T$ where we recall that $\lambda$ is defined by $1 / R=\lambda \epsilon^{\frac{7}{4}}$ where $R$ is the Reynolds number. We are in a régime where $\lambda \ll 1$ but the time is very large compared to the diffusion time in the critical-layer, that is $T \gg 1 / \lambda$, and so $\sqrt{\lambda T} \gg 1$; thus we cannot neglect this quantity. In this section, we will examine this situation and explicitly express the additional mean flow $U_{1}$ as a function of $\lambda T$ and $Y$.

We will use a theory of the diffusion boundary layer developed by Troitskaya and Reznik (1996) who applied it to the quasi-steady critical layer of a stratified shear flow. In spite of the different scalings here, the resulting equations are the same, and so we will display their equations without giving details.

Essentially, a fourth variable is now used, the diffusive wavenumber $\nu=1 /(2 \sqrt{\lambda T})$ which is very small. The general motion of the critical layer is given by

$$
y_{c}=y_{c 0}+\epsilon^{\frac{1}{2}} 2 \kappa(\epsilon, \nu) \sqrt{\lambda T} .
$$

Here $\kappa$ is an unknown function of $\nu$ that tends to a constant when $\epsilon^{\frac{1}{2}} \sqrt{\lambda T} \rightarrow 0$. $y_{c 0}$ is the critical level in the limit when $\lambda T \rightarrow 0$. The inner cross-stream variable $Y$ is thus a function of $\nu$. The nonlinear critical layer analysis in section 4 must now take into account the evolution in a moving critical layer. This also implies a slowly evolving wave speed: $c(\nu)$; indeed the critical level is now determined by the condition

$$
U\left(y_{c}\right)=c+T \partial_{T} c=c-\frac{1}{2} \nu \partial_{\nu} c .
$$

The wave speed is easily obtained through an expansion in $\epsilon^{\frac{1}{2}} / \nu$

$$
c=U_{0}\left(y_{c 0}\right)+\frac{2}{3} \frac{\epsilon^{\frac{1}{2}}}{\nu} \kappa(\epsilon, \nu) U_{0}^{\prime}\left(y_{c 0}\right)+\epsilon^{\frac{1}{2}} U_{1}\left(y_{c 0}\right)+\ldots \quad \text { provided that } \quad \lambda T \ll 1 / \epsilon .
$$

\subsection{The diffusion boundary layer}

Here, we examine the outer flow in the region far from the critical layer but where $Y \sim 1 / \nu$, so a relevant inner scaling is

$$
H=\nu Y .
$$

The related streamfunction is $\Psi_{D B}=\nu^{2} \Psi$. Using this scaling, we find that the mean vorticity equation in the diffusion boundary (DB) layer, (cf. Troitskaya and Reznik (1996)), at the lowest order is

$$
Q_{D B, H H}^{(2)}+2 \nu Q_{D B, \nu}^{(2)}+2\left(H+\kappa-\nu \frac{d \kappa}{d \nu}\right) Q_{D B, H}^{(2)}=\frac{1}{\lambda} \mathcal{F}_{m, H H}
$$


with $Q_{D B}^{(2)}=\Psi_{D B, Y Y}^{(2)}$. The momentum flux $\mathcal{F}_{m}$ is assumed to be constant outside of the critical layer. It follows, from matching with the outer flow, that here the right-hand side is zero. The boundary conditions are obtained by matching the DB layer with the undisturbed flow $U_{u}(y)$ as $|H| \rightarrow \infty$ and with the critical layer at $H=\nu Y$; as $Y=O(1)$, so $H=O(\nu)$ and at the zeroth order in $\nu, H=0$.

The determination of the leading-order flow $\left(Q_{D B}^{(2)}, U_{D B}^{(2)}\right)$ within the diffusive boundary layers is performed in Appendix D. The main outcome is that an additional undisturbed mean shear flow $\epsilon^{\frac{1}{2}} U_{u, 1}$ is indispensable for our present theory. Since $U_{0}$ is stationary due to the forcing balancing its dissipation, it follows that $U_{1}$ in the critical layer cannot be created by the diffusion of $U_{0}$ but is due to an additional flow that, away from the critical layer remains in an undisturbed state, $U_{u, 1}$ say. This implies that we have a new stationary flow $U_{\text {new }}=U_{0}-\epsilon^{\frac{1}{2}} U_{u, 1}$ connected to the forcing $F_{\text {new }}=-\lambda U_{\text {new }}^{\prime \prime \prime}$. So, the undisturbed flow becomes $U_{u}=U_{\text {new }}+\epsilon^{\frac{1}{2}} U_{u, 1}$; by diminishing $F$, we allow the diffusion of the mean flow $U_{u, 1}$ around the critical layer to create the flow $U_{1}$. This new body force does not alter the amplitude equation. The same development can be carried out at the next order for $Q_{D B}^{(5)}=\Psi_{D B, Y Y}^{(5)}$. As the $O\left(\epsilon^{2}\right)$ vorticity cannot be matched on the separatrix, we do not know the jump of $U_{2}^{\prime}$, although we can find a single relationship linking the jumps of $U_{2}^{\prime}$ and $U_{2}^{\prime \prime}$ obtained at $O\left(\epsilon^{\frac{5}{2}} \ln \epsilon\right)$. This degree of freedom allows us to choose the value of $\left[\alpha_{l, 2}\right]_{-}^{+}$(cf. its expression (57)).

\subsubsection{Effect on the critical-layer flow}

Equation (25) is modified by the movement of the critical level in such a way that its right-hand side becomes at the leading order

$$
\epsilon \lambda\left\{\partial_{Y}^{4} \Psi+2 \nu \epsilon^{\frac{1}{2}}\left[\nu^{2} \partial_{Y}^{2} \Psi_{\nu}+\left(\kappa-\nu \kappa_{\nu}\right) \partial_{Y}^{3} \Psi\right]\right\}
$$

The first modification within the bracket occurs for the $O\left(\epsilon^{2}\right)$ flow and is of order $\nu$. It is thus negligible compared to the previous terms that depend on $\epsilon^{\frac{1}{2}} / \nu$. The amplitude equation is hence not modified by the diffusion of the outer flow.

\section{The amplitude equation}

The amplitude $A$ satisfies the following equation

$$
\partial_{T} A+R_{0} A \partial_{X} A+S_{0} \partial_{X}^{3} A+V_{0} \partial_{X} A=\mathcal{C}_{X}[A] .
$$

In general, due to the right-hand side, it would seem likely that this equation is not integrable, and needs to be solved numerically. However, it is possible to find a travelling solitary wave explicitly. Thus, we seek a solution where $A=A(\zeta), \zeta=X-V T$ where $A \rightarrow 0$ as $\zeta \rightarrow \pm \infty$, so that (69) becomes

$$
\left(V_{0}-V\right) A+\frac{1}{2} R_{0} A^{2}+S_{0} \partial_{X}^{2} A=\mathcal{C}[A] .
$$

Next, using the expression (50) for $\mathcal{C}[A]$, together with $(46,47$ and 48$)$, we get

$$
\begin{gathered}
-V A+4 \mu r_{0} A^{2}+\varpi\left(Y_{s}+Y_{\infty}\right) A+\mu M(A)=\chi\left(Y_{s}+Y_{\infty}\right) \partial_{X}^{2} A, \\
\text { where } Y_{s}=\left[2\left(A_{0}-A\right)\right]^{\frac{1}{2}}, \quad Y_{\infty}=\sqrt{2 A_{0}}, \\
\text { and } M(A)=\left\{2 A_{0}\left(a-1-\frac{4}{9} \hat{\beta}\right)-Y_{s} Y_{\infty}\left(1-a+\frac{5}{9} \hat{\beta}\right)\right\} A, \\
\text { while } \quad \chi=-\frac{1}{4}\left[\alpha_{d, 1}\right]_{-}^{+} / \bar{b}_{0}, \varpi=\frac{1}{4}\left[\alpha_{l, 1}\right]_{-}^{+} / \bar{b}_{0}, \mu=\frac{1}{2} b_{0} U_{0}^{\prime}, R_{0}=8 \mu r_{0} .
\end{gathered}
$$

Here we recall that $A_{0}=A(0)$. This can readily be integrated once more to yield

$$
\begin{aligned}
\chi\left(\partial_{X} \hat{A}\right)^{2}=\mu Y_{\infty}(1-\hat{A})\left\{\left(\Omega-\frac{14}{5} r_{0}\right)\left[\frac{2}{3}(1-\hat{A})^{\frac{1}{2}}-1\right]-2 r_{0}\left[1+\hat{A}-\frac{4}{5}(1-\hat{A})^{\frac{1}{2}}\left(\frac{2}{3}+\hat{A}\right)\right]\right. \\
\left.-\Omega(1-\hat{A})^{\frac{1}{2}}\left[(1-\hat{A})^{\frac{1}{2}}-\frac{4}{3}\right]\right\}
\end{aligned}
$$


A

(a)

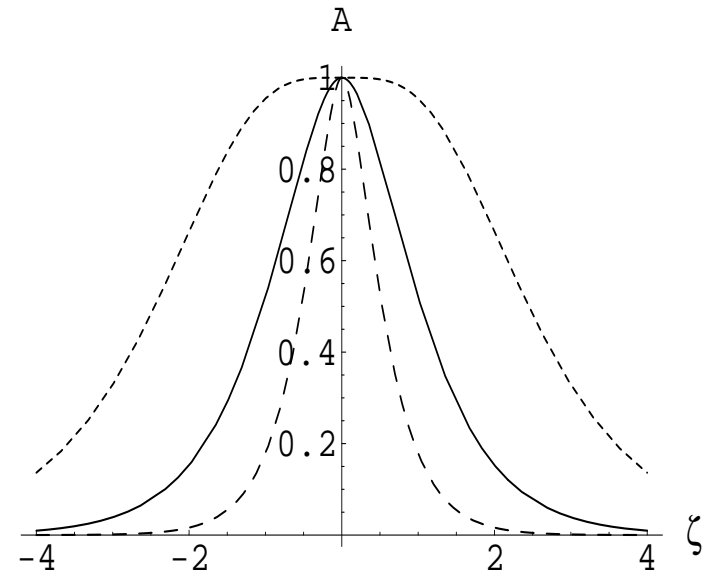

(b)

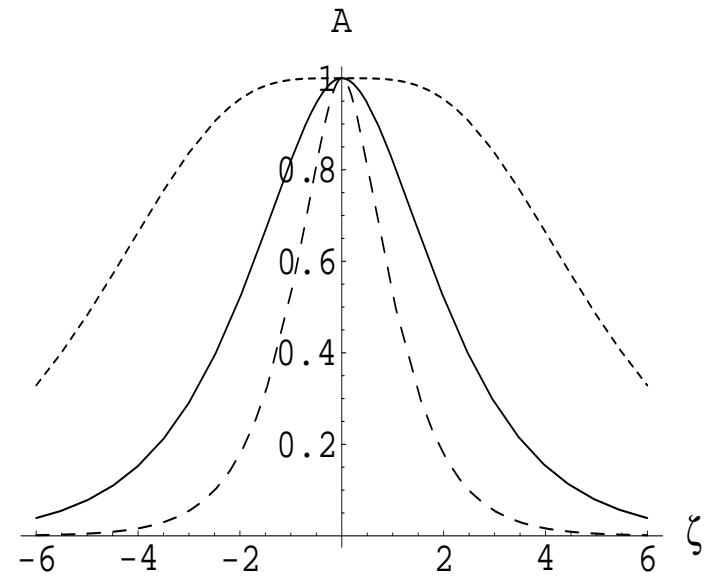

Figure 2: (a) $\hat{A}$ for $r_{1}=0,-1,-5$ (dotted line, solid line and dashed line) $\zeta_{0}=1$, (b) $\hat{A}$ with $\zeta_{0}=2$ and the same values of $r_{1}$ When $r_{1} \rightarrow-\infty, \hat{A}(\zeta)$ tends to the Dirac- $\delta$ function.

$$
\text { where } \Omega=1-a+\frac{5}{9} \hat{\beta}-\frac{1}{2} \frac{\left[\alpha_{l, 1}\right]_{-}^{+}}{b_{0}^{2} Y_{\infty}} \text {, and } \hat{A}=\frac{A}{A_{0}} \text {. }
$$

The speed is given by

$$
V=\mu\left(a+\frac{9}{5}-\frac{1}{45} \hat{\beta}\right) A_{0}+\frac{1}{2} \varpi Y_{\infty} .
$$

We re-arrange (72) by putting $Y_{s}^{*}=(1-\hat{A})^{\frac{1}{2}}$

$$
\left(\partial_{X} \hat{Y}_{s}\right)^{2}=\frac{4}{5} r_{0} \frac{b_{0}^{2} Y_{\infty}}{\left[\alpha_{d, 1}\right]_{-}^{+}}\left(Y_{s}^{* 3}+\frac{5}{8}\left(\frac{\Omega}{r_{0}}-2\right) Y_{s}^{* 2}-\frac{5}{4}\left(\frac{2}{5}+\frac{\Omega}{r_{0}}\right) Y_{s}^{*}+\frac{3}{4}+\frac{5}{8} \frac{\Omega}{r_{0}}\right) .
$$

This can be reduced to a degenerate elliptic equation

$$
\begin{gathered}
\int_{0}^{Y_{s}^{*}} \frac{d r}{\sqrt{s_{0}\left(r-r_{1}\right)}(1-r)}= \pm \frac{\zeta}{\zeta_{0}} \\
\text { where } \zeta_{0}=\left|\frac{5}{4} \frac{\left[\alpha_{d, 1}\right]_{-}^{+}}{b_{0}^{2} r_{0} Y_{\infty}}\right|^{\frac{1}{2}}, r_{1}=-\frac{3}{4}-\frac{5}{8} \frac{\Omega}{r_{0}} \text { and } s_{0}=\operatorname{sign}\left[r_{0}\right] .
\end{gathered}
$$

Note that $\left[\alpha_{d, 1}\right]_{-}^{+}$is always positive. If $s_{0}=1$ then we require that $r_{1}<0$, or $\Omega / r_{0}>-6 / 5$, and then

$$
\hat{A}=1-\left(r_{1}+\left(1-r_{1}\right) \tanh ^{2}\left\{\operatorname{arctanh}\left[\left(\frac{-r_{1}}{1-r_{1}}\right)^{\frac{1}{2}}\right]+\frac{1}{2}\left(1-r_{1}\right)^{\frac{1}{2}}\left|\frac{\zeta}{\zeta_{0}}\right|\right\}\right)^{2} .
$$

On the other hand if $s_{0}=-1$ then we require that $r_{1}>1$, or $\Omega / r_{0}<-14 / 5$ and then

$$
\left.\hat{A}=1-\left(r_{1}+\left(1-r_{1}\right) \operatorname{cotanh}^{2}\left\{\operatorname{arctanh}\left[\left(\frac{r_{1}-1}{r_{1}}\right)^{\frac{1}{2}}\right]+\frac{1}{2}\left(r_{1}-1\right)^{\frac{1}{2}}\left|\frac{\zeta}{\zeta_{0}}\right|\right]\right\}\right)^{2} .
$$

Note that this case holds when $r_{0}<0$, which in turn requires that $8 / 9 \beta<U_{0}^{\prime \prime}<\beta$, which is quite a restrictive condition. For both waves, the width of the hump is inversely proportional to $A_{0}^{\frac{1}{4}}$. The solution related to the case $r_{0}=0$, that is (1) without the quadratic term is defined by

$$
\hat{A}=1-\left(1-\exp \left[-\frac{\left|b_{0}^{2} Y_{\infty} \nu \zeta\right|}{2\left[\alpha_{d, 1}\right]_{-}^{+}}\right]\right)^{2}
$$

Some typical plots of these waves and the corresponding separatrices are shown in figures 2,3 and 4 . 
(a)

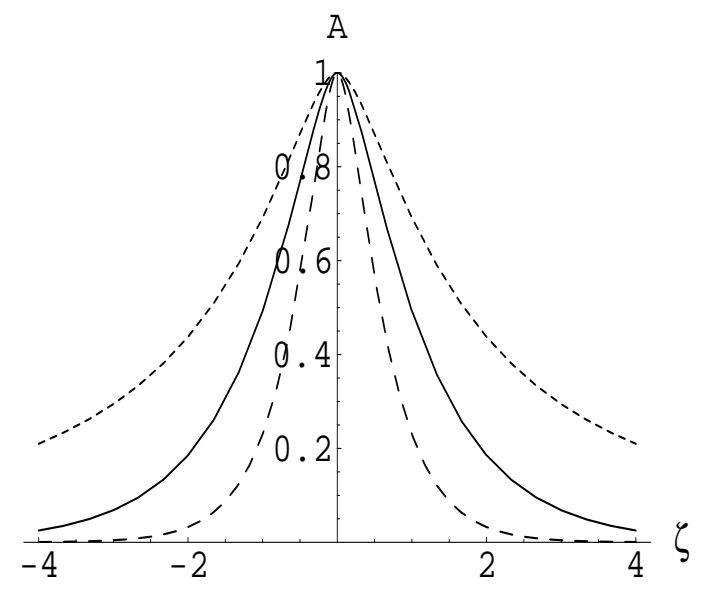

Figure 3: (a) $\hat{A}$ for $r_{1}=1,2,5$ (as in figure 2 ) and $\zeta_{0}=1 . r_{1} \rightarrow 1, \hat{A} \rightarrow 1-\left[\left|\frac{\zeta}{\zeta_{0}}\right| /\left(2+\left|\frac{\zeta}{\zeta_{0}}\right|\right)\right]^{2}, r_{1} \rightarrow \infty$, $\hat{A}(\zeta)$ tends to the Dirac- $\delta$ function.

(a)

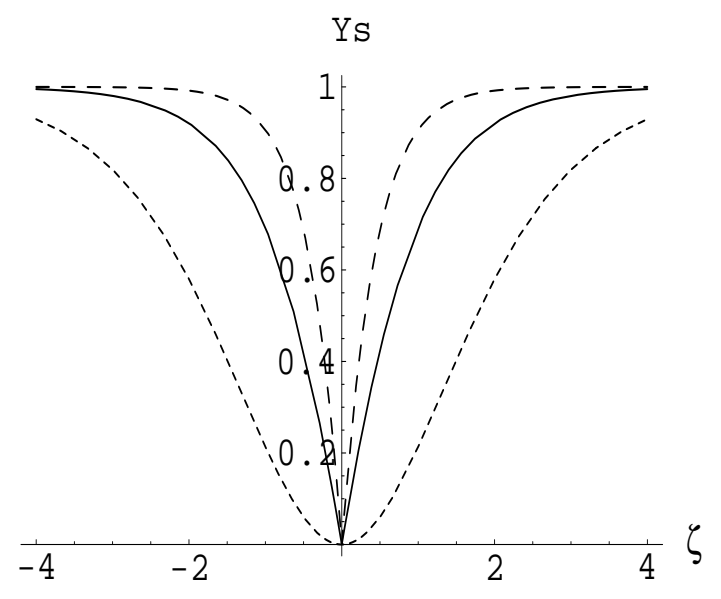

(b)

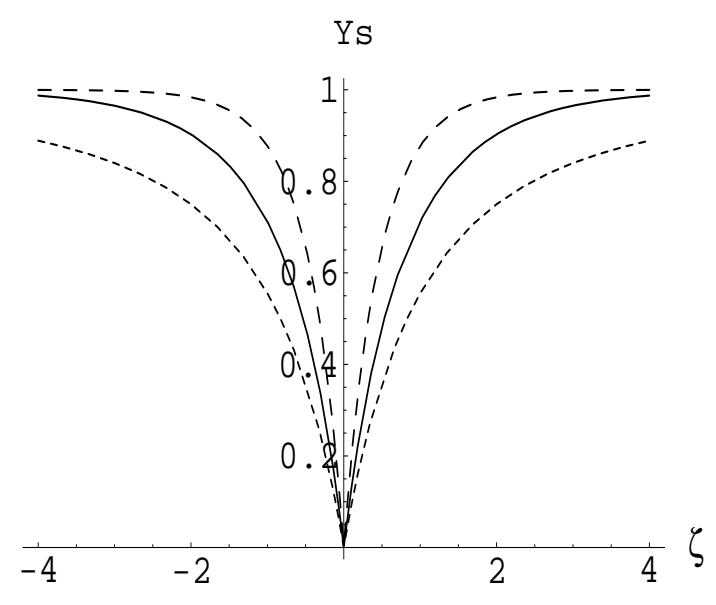

Figure 4: (a) $\hat{Y}_{s}$ for this case $2, d Y_{s} / d \zeta(0)$ is proportional to $\left(-r_{1}\right)^{\frac{1}{2}}$; (b) $\hat{Y}_{s}$ for case $3(\mathrm{a}), d Y_{s} / d \zeta(0)$ is proportional to $\left(r_{1}\right)^{\frac{1}{2}}$ 


\section{Nonlinear critical layer neutral modes}

We now consider the issue of the existence of such solitary-wave motions coupled with a nonlinear critical layer, as described in the preceding sections. The problem reduces to finding a solution $\phi(y)$ of the Rayleigh-Kuo equation (12) in the form (15), that satisfies the boundary conditions (16) in the outer flow far from the critical layer, and matches the local Frobenius solutions (13) at the edges of the critical layer. Further, in order to have a solitary wave solution, we must also have $r_{1} \leq 0$ or $r_{1} \geq 1$ (see (74). Note that for a finite-period nonlinear wave, a pair $\left(c_{0}, k\right)$ may exist that satisfies the first two conditions, with $\beta$ being a given parameter. It is the singular neutral mode. But, for $k$ tending to zero, only some values of $\beta$ may be allowed. The solitary wave condition on $r_{1}$ may be a further restriction depending on the chosen velocity profile $U_{0}$. The numerical integration of the Rayleigh-Kuo equation is necessary as the boundary conditions (16) are usually not located near the critical layer. Thus $\phi=a b_{0} \phi_{a}+\phi_{b}$ is computed using a fourth-order Runge-Kutta scheme in each of the upper and lower regions of the outer flow. $\phi_{a}$ and $\phi_{b}$ are computed a second time via the Frobenius local solutions at the levels $y=y_{c} \pm \delta$, where $\delta$ is a measure of the width of the critical layer and will be taken as $\delta=10 \times h$, where $h$ is the integration step. The coefficient $a$ is determined from matching both solutions at $y=y_{c}-\delta$ by cancelling the Wronskian of numerical and local solutions. $\beta$ is then computed by using a secant method finding the root of the Wronskian at $y=y_{c}+\delta$.

For the additional undisturbed profile $U_{u, 1}(y)$, we choose shape perturbation of the flow $U_{0}$; that is, for instance, if we study the hyperbolic tangent mixing layer, $U_{u, 1}$ will be also defined by $\tanh y$ but multiplied a coefficient so that $U_{u, 1}^{\prime}\left(y_{c 0}\right)=U_{u, 1}^{\prime}$. The given parameters are $y_{c 0}$ and $U_{u, 1}^{\prime}$; as all mean-flow jumps like $\left[U_{1}^{\prime}\right]_{-}^{+},\left[U_{1}^{\prime \prime}\right]_{-}^{+} \ldots$ are proportional to $Y_{\infty},\left[\alpha_{l, 1}\right]_{-}^{+}$is also proportional to $Y_{\infty}$ and the latter is used to non-dimensionalize. We will henceforth prefer employing the ratio $\sigma=U_{u, 1}^{\prime} /\left(b_{0} U_{0}^{\prime} Y_{\infty}\right)\left(b_{0}\right.$ and $U_{0}^{\prime}$ evaluated in $\left.y_{c 0}\right)$ as the second parameter. $\kappa_{0}$ is determined from (D9), and then all parameters describing the diffusion layer, $q_{11}^{ \pm}, u_{10}, U_{u, 1}^{\prime \prime}, \kappa_{1} \ldots$ can be found. Thus, we can then compute $y_{c}$ and the Frobenius series coefficients, $a_{0, n}$ and $b_{0, n}$. The Cauchy part of the integral in (20) is computed by separating the different outer flows, namely, the undisturbed flow, the diffusion layer and the flow around the critical layer described by the Frobenius solutions. The DB layers are asymmetrical vis-à-vis the critical layer; the edges of the diffusion layers are defined by the intersections between the undisturbed and DB velocity curves. These connections are not always possible, and depend on the choices of $U_{0}(y)$ and $U_{1}(y)$. This is a third limitation (denoted by L3) to the existence of the neutral modes, the first limitation being the long-wavelength assumption (L1) and the second condition (L2) being the solitary-wave requirement that $r_{1}<0$ or $r_{1}>1$. Another limitation also arises due to the proximity of the boundaries; however, here the wall and diffusion layers interaction was not examined owing to its complexity. In addition, we have another limitation (L4) due to the strong truncation to the first order for $\kappa$.

The velocity profiles studied are:

- (a) Poiseuille flow: $U_{0}(y)=U_{0}^{\prime \prime} / 2\left(y^{2}-d^{2}\right)$ for $0 \leq y \leq d$; we deliberately avoid the interaction of two critical layers by taking $y \geq 0$;

- (b) A jet profile: $U_{0}(y)=\operatorname{sech}^{2}(y)$ for $0 \leq y \leq d$ with the same remark;

- (c) A laminar mixing layer: $U_{0}(y)=\tanh (y)$ for $-d \leq y \leq d$.

$d$ is chosen large for (b) and (c) so that the flow at $y \sim|d|$ is nearly uniform. The boundary conditions are that $\phi=0$ at $y=0, d$ for (a), $\phi=0$ at $y=0$ for (b), $\phi$ decays exponentially at $y=d$ for (b) and at $y=-d$ for $(\mathrm{c})$, and $\phi$ radiates at $y=d$ for $(\mathrm{c})$.

We have plotted the curves $c_{0}$ as a function of $\beta$ for different values of the ratio $\sigma$. In Figure 5 (a), we examine the effect of $\sigma$ on the neutral modes for a Poiseuille velocity profile. When $\sigma=-1$, we observe two branches at $\beta \simeq 0$ and $\beta \simeq 10$. Other branches not shown in the graph exist for larger $\beta$ $(\beta \simeq 28,54,88 \ldots)$. Both branches support solitary waves. When $\sigma<0, U_{0}(y)$ and $U_{1}(y)$ have opposite signs. Decreasing the mean velocity seems to have a favorable effect on the existence of solitary waves. Indeed, no modes were found when $\sigma=1$ whatever the branch may be, L3 being never satisfied. In Figure (6), the hyperbolic-tangent profile neutral modes are displayed for $\sigma=1$ and $\sigma=-2$. Two branches exist. One for small values of $\beta(\beta \leq 0.4)$ and another which may span all possible range of $\beta$. However, for large $\beta,\left|\kappa_{1}\right|$ is also large. As a result, our truncated first-order expansion in $\epsilon^{\frac{1}{2}} / \nu$ is insufficient and we are hence restricted to a maximal $\beta \sim 2$. Both branches are invariant when $\sigma$ varies but the permittive range of $\beta$ changes. When the first branch is nearly forbidden, we found only two non-converging modes. 
(a)

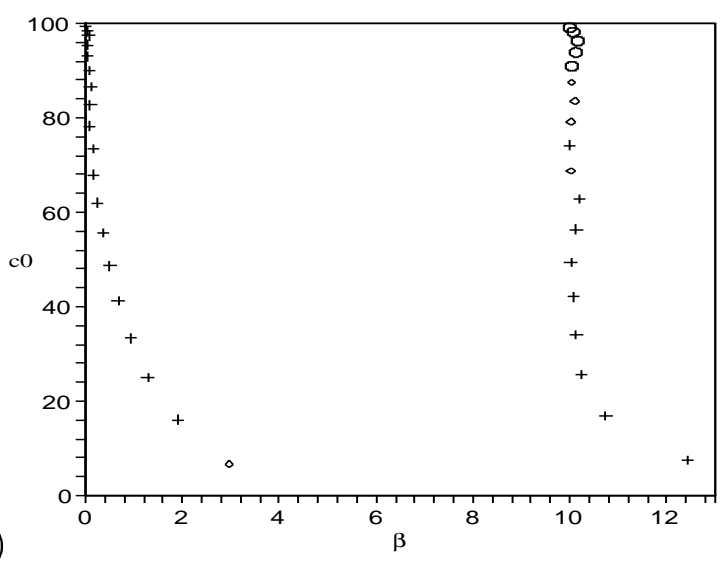

(b)

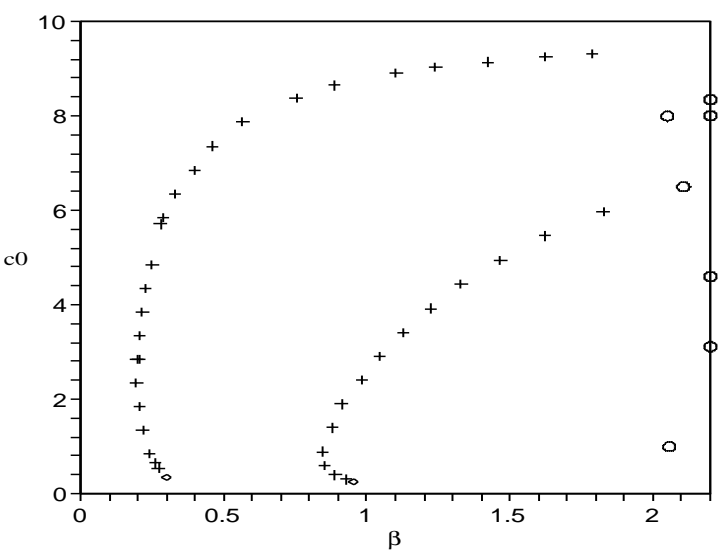

Figure 5: Neutral modes $(d=10)$ : (a) Poiseuille flow: $U_{0}^{\prime \prime}=-2, \sigma=U_{u, 1}^{\prime} /\left(b_{0} U_{0}^{\prime} Y_{\infty}\right)=-1, \kappa_{0}=0.5419$, (b) Couette flow, $U_{0}^{\prime}=1, \sigma=1 ;+$ denotes modes supporting a solitary wave (L2+L3+L4), $\diamond$ denotes modes not supporting a solitary wave (but satisfying L3+L4), $\square$ denote modes supporting a solitary wave but the $\epsilon^{\frac{1}{2}} / \nu$ series is not convergent (L2+L3), o denotes modes not satisfying L3.

(a)

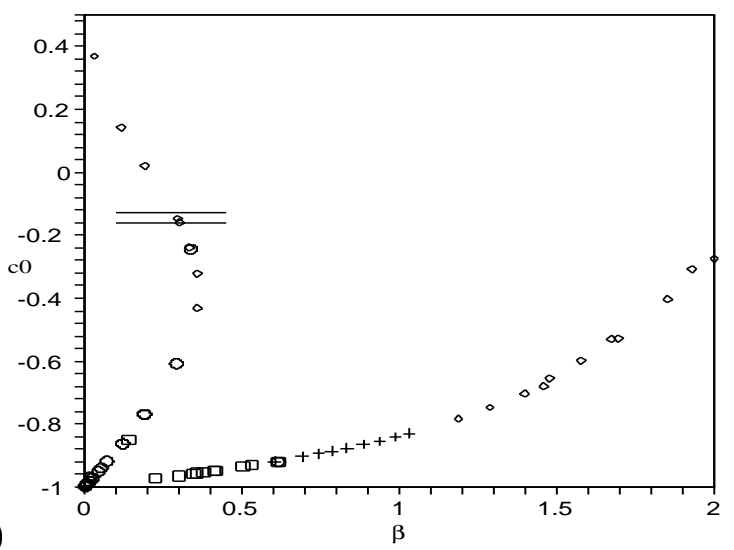

(b)

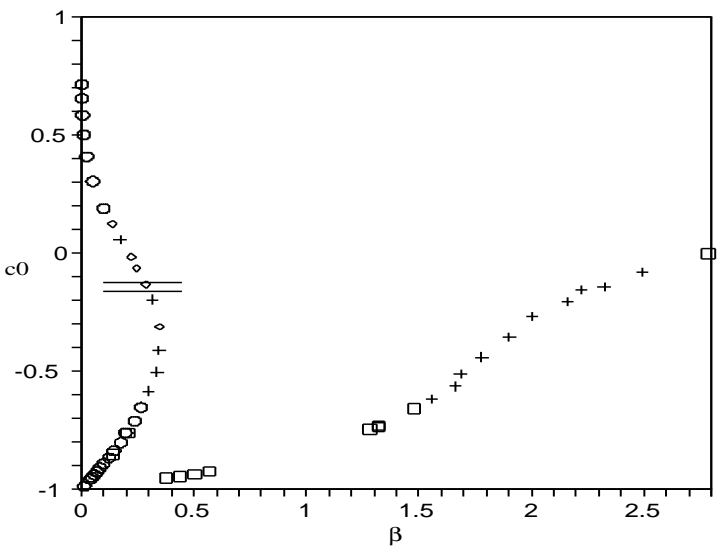

Figure 6: Neutral modes within a mixing-layer flow: $d=10$ (a) $\sigma=1, \kappa_{0}=-0.5419$, (b) $\sigma=-2$, $\kappa_{0}=0.2790 ;$ Both short straight lines bound the domain of the D-wave with $r_{1} \geq 1$. + denotes modes supporting a solitary wave (L2+L $3+\mathrm{L} 4), \diamond$ denotes modes not supporting a solitary wave (but satisfying L3+L4), $\square$ denotes modes supporting a solitary wave but the $\epsilon^{\frac{1}{2}} / \nu$ series is not convergent (L2+L3), denotes modes not satisfying L3. 
(a)

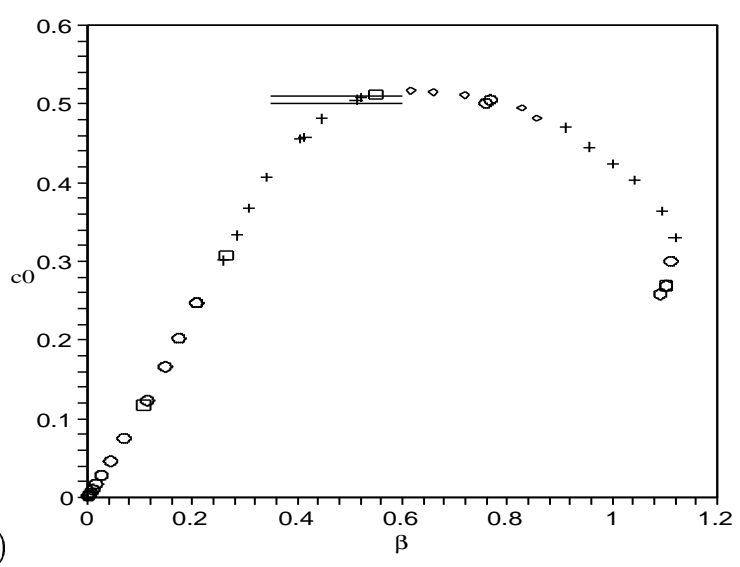

(b)

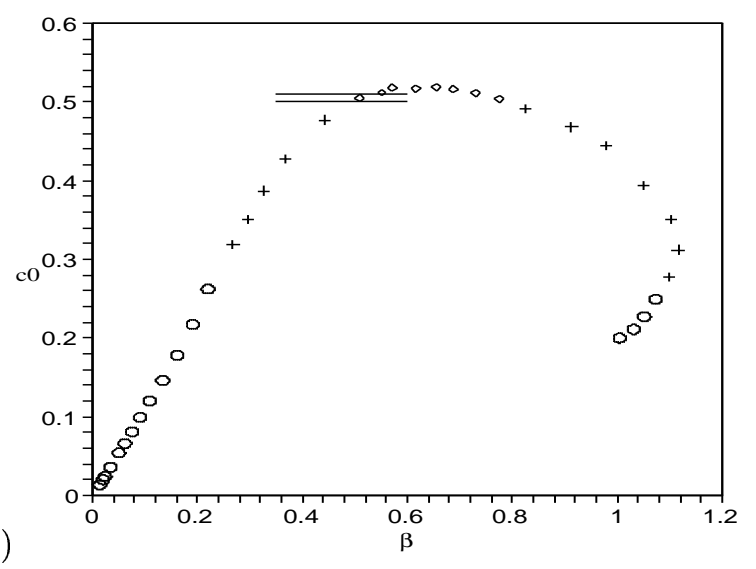

Figure 7: Neutral modes within a jet flow: $d=10$ (a) $\sigma=1, \kappa_{0}=-0.5419$, (b) $\sigma=2, \kappa_{0}=-0.2790$. Both short straight lines bound the domain of the D-wave with $r_{1} \geq 1$. + denotes modes supporting a solitary wave (L2+L3+L4), $\diamond$ denotes modes not supporting a solitary wave (but satisfying L3+L4), denotes modes supporting a solitary wave but the $\epsilon^{\frac{1}{2}} / \nu$ series is not convergent (L2+L3), o denotes modes not satisfying L3.

Whereas on the second branch, the modes are located between $\beta=0$ and $\beta \simeq 1.1$ for $c_{0}$ varying between -1 and -0.8 . The expansion does not converge rapidly for $c_{0}$ close to -1 . When $\sigma=-2$, modes are present on the first branch for $-0.6<c_{0}<0.1$; for the second branch, the expansion strongly diverges and so many truncated modes are not located on the branch. It is the reason why a gap lies between $\beta \simeq 0.6$ and $\beta \simeq 1.2$. A negative $\sigma$ favors the existence of modes. In Figures (7), the jet profile gives one unique branch the same for all $\sigma$ that has a maximal $c_{0} \simeq 0.5$ and a maximal $\beta \simeq 1.1$. The curve around the maximal $c_{0}$ does not permit a solitary wave mode (L2). The computation for $\sigma=2$ seems to converge more rapidly than for $\sigma=1$. The second difference is the range where solitary wave modes are forbidden. For the first curve, the range is between $\beta \simeq 0.6$ and 0.9 whereas for the second curve, it is between $\beta \simeq 0.5$ and 0.8 . The ends of the branch are characterized by modes not satisfying (L3). The areas where the modes should satisfy (L2) with $r_{1} \geq 1$ are delimited by short straight lines on the graphs, and they are very small. For instance, in Figure 7 (a) the modes $\left(\beta=0.521, c_{0}=0.508\right)$ and ( $\left.\beta=0.512, c_{0}=0.505\right)$ are such as $r_{1}<0$ and are located at the frontier between the modes that do or do not satisfy (L2). In figure 8 (a), (L4) is not satisfied for $r_{1} \leq 0 . \sigma<0$ decreases the $\beta$-range of the solitary-wave neutral modes, Figure 8 (a) shows again that for $\sigma=-2$, these neutral modes are confined around two small zones with $\beta \simeq 0.4-0.5$ and 0.8 . This should be contrasted with the corresponding results for the mixing-layer profile. These few examples thus show the importance of the choice of $U_{0}$. Figure 8 (b) displays the behaviour of $U_{1}(y)$ in the outer flow. The two different velocity gradients at either side of the critical layer are clearly seen. However, the connections between the diffusion layers and the undisturbed flow are not distinguishable, which is no longer true when the mode approaches a zone where (L3) is not satisfied.

This section demonstrates that whatever the velocity profile $U_{0}(y)$, it is possible to find an undisturbed flow $U_{u, 1}$ such that the critical layer could diffuse and adjust the vorticity on its edges. The number of modes supporting solitary waves is infinite although belonging to small sets in $\beta$. Except for the Poiseuille flow, the relevant $\beta$ does not exceed an order one value.

\section{The case of a linear shear flow}

Here we assume that the mean flow obeys is given by $U_{0}(y)=c+U_{0}^{\prime} \eta$, that is a Couette flow, for which case we can obtain some explicit formulae. Since now $r_{0}=8 / 9>0$, this constant-shear flow only permits the first case (75). We display here the coefficients of the amplitude equation and then explicitly give the 
(a)

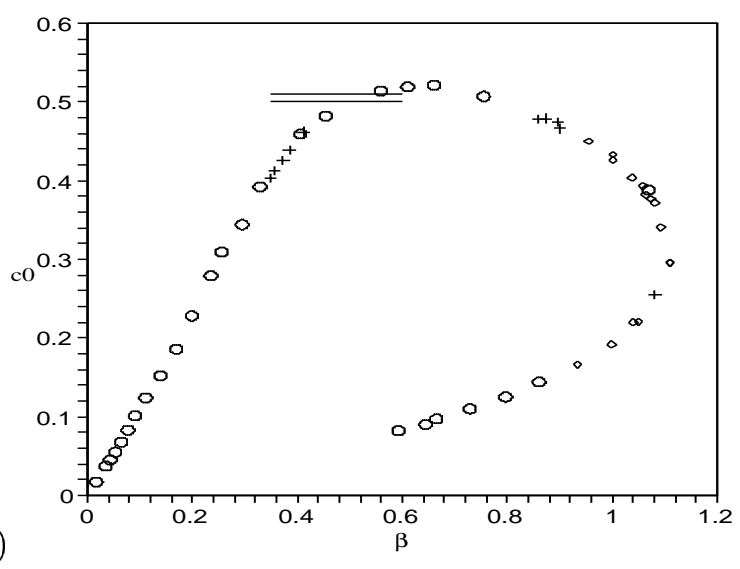

(b)

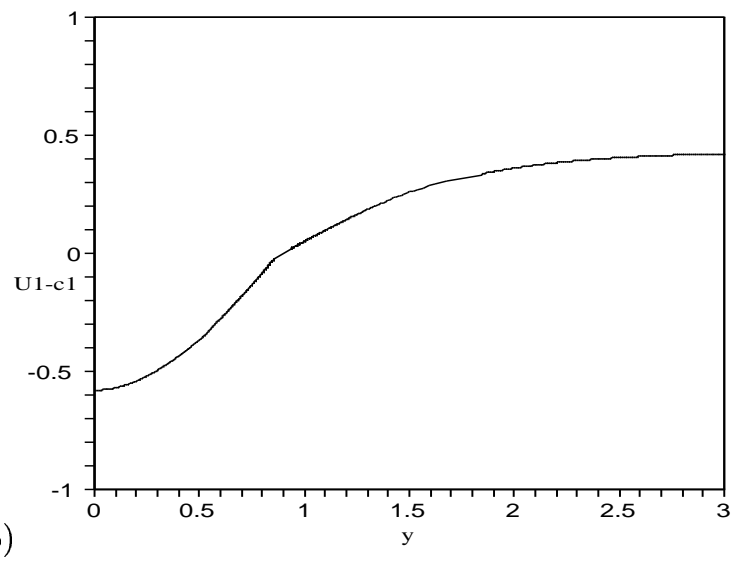

Figure 8: Neutral modes within a jet flow: $d=10$ (a) $\sigma=-2, \kappa_{0}=0.2790$. Both short straight lines bound the domain of the D-wave with $r_{1} \geq 1$. + denotes modes supporting a solitary wave (L2+L3+L4), $\diamond$ denotes modes not supporting a solitary wave (but satisfying L3+L4), $\square$ denotes modes supporting a solitary wave but the $\epsilon^{\frac{1}{2}} / \nu$ series is not convergent (L2+L3), o denotes modes non satisfying L3. (b) $\mathrm{U} 1(\mathrm{y})$-c1, with $\sigma=2, \beta=0.826, c=0.491, y_{c}=0.894, y_{c 0}=1$ and $a=-0.385 ; U_{D B}^{(1)}$ intersects $U_{u, 1}$ at $y=0.524$ and $y=1.844$.

expression of the streamfunction at the first three orders.

$$
\begin{gathered}
V_{0}=\left(\frac{5}{9}-a\right) \beta S_{c}-\frac{1}{4}\left[\alpha_{l, 1}\right]_{-}^{+} \frac{Y_{\infty}}{\bar{\beta}}, \quad R_{0}=-\frac{32}{9} \beta, \quad S_{0}=-\frac{1}{4}\left[\alpha_{d, 1}\right]_{-}^{+} \frac{Y_{\infty}}{\bar{\beta}}, \\
\mathcal{C}[A]=\frac{1}{4}\left\{\left[\alpha_{l, 1}\right]_{-}^{+} A+\left[\alpha_{d, 1}\right]_{-}^{+} \partial_{X}^{2} A\right\} \frac{Y_{s}}{\overline{\bar{\beta}}}-\frac{1}{2} \beta Y_{\infty}\left(\frac{4}{9}-a\right) A Y_{s}, \quad V=-\left(\frac{1}{2} a+\frac{41}{45}+\frac{1}{4} \frac{\left[\alpha_{l, 1}\right]_{-}^{+}}{\bar{\beta}^{2} Y_{\infty}}\right) \beta S_{c} .
\end{gathered}
$$

Note that $R_{0}$ and $S_{0}$ are always negative.

\subsection{The singular mode}

This can be expressed in terms of the dimensionless coordinate $\varrho=2|\bar{\beta} \eta|^{\frac{1}{2}}$ and the Bessel functions $J_{1}$ and $Y_{1}$ of the first kind.

$$
\begin{aligned}
\phi^{+}(y) & =\varrho\left[\mathcal{A}^{+} J_{1}(\varrho)+\mathcal{B}^{+} Y_{1}(\varrho)\right], \quad \text { if } \quad \bar{\beta}>0, \\
\phi^{-}(y) & =\varrho\left[\mathcal{A}^{-} J_{1}(i \varrho)+\mathcal{B}^{-} Y_{1}(i \varrho)\right], \\
& \\
\phi^{+}(y) & =\varrho\left[\mathcal{A}^{+} J_{1}(i \varrho)+\mathcal{B}^{+} Y_{1}(i \varrho)\right], \quad \text { if } \quad \bar{\beta}<0, \\
\phi^{-}(y) & =\varrho\left[\mathcal{A}^{-} J_{1}(\varrho)+\mathcal{B}^{-} Y_{1}(\varrho)\right] .
\end{aligned}
$$

Henceforth, we will only describe the first case; the other case is similar. Near $y_{c}$

$$
\begin{aligned}
& \phi^{+}(y)=-\frac{2}{\pi}\left\{\mathcal{B}^{+} \phi_{b}+b_{0}\left[\pi \mathcal{A}^{+}+\mathcal{B}^{+}(2 \gamma-1+\ln \varphi)\right] \phi_{a}\right\}, \\
& \phi^{-}(y)=i \frac{2}{\pi}\left\{\mathcal{B}^{-} \phi_{b}+b_{0}\left[\pi \mathcal{A}^{-}+\mathcal{B}^{-}(2 \gamma-1+\ln -\varphi)\right] \phi_{a}\right\}, \quad \text { with } \varphi=\bar{\beta} \eta_{0} .
\end{aligned}
$$

Here $\gamma$ is the Euler constant. By equating this expansion with (15), we have $\mathcal{B}^{+}=-\pi / 2$ and $\mathcal{B}^{-}=-i \pi / 2$. The boundary conditions (16) yield

$$
\mathcal{A}^{+}=\frac{\pi}{2} \frac{Y_{1}\left(\varrho_{2}\right)}{J_{1}\left(\varrho_{2}\right)}, \quad \mathcal{A}^{-}=i \frac{\pi}{2} \frac{Y_{1}\left(i \varrho_{1}\right)}{J_{1}\left(i \varrho_{1}\right)}
$$



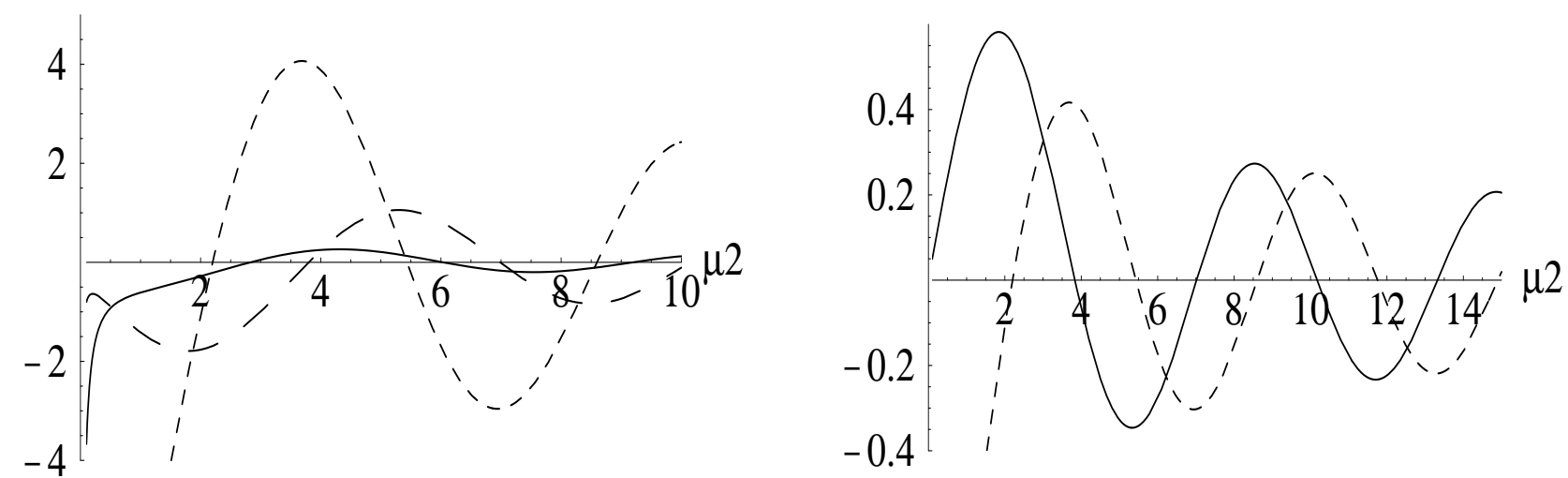

(a)

(b)

Figure 9: (a) $\Im\left[J_{1}\left(i \varrho_{1}\right) Y_{1}\left(\varrho_{2}\right)-J_{1}\left(\varrho_{2}\right) Y_{1}\left(i \varrho_{1}\right)\right]$ as a function of the dimensionless coordinate $\varrho_{2}: \varrho_{1}=0.2$ - - $\varrho_{1}=1$ - and $\varrho_{1}=4--$; (b) $J_{1}\left(\varrho_{2}\right)-, Y_{1}\left(\varrho_{2}\right)--, j_{1,1}=3.832, j_{1,2}=7.016, j_{1,3}=10.174$, $y_{1,1}=2.197, y_{1,2}=5.430$ and $y_{1,3}=8.596$.

$$
\text { and } \quad a^{+}=-1+2 \gamma+\ln \varphi-\pi \frac{Y_{1}\left(\varrho_{2}\right)}{J_{1}\left(\varrho_{2}\right)}, \quad a^{-}=-1+2 \gamma+\ln -\varphi-\pi \frac{Y_{1}\left(i \varrho_{1}\right)}{J_{1}\left(i \varrho_{1}\right)} .
$$

The condition for a zero phase jump gives the dispersion relation

$$
\frac{Y_{1}\left(\varrho_{2}\right)}{J_{1}\left(\varrho_{2}\right)}-\frac{Y_{1}\left(i \varrho_{1}\right)}{J_{1}\left(i \varrho_{1}\right)}=-i
$$

To solve this relation numerically, we must examine the roots of $\Im\left[Y_{1}\left(\varrho_{2}\right) J_{1}\left(i \varrho_{1}\right)-Y_{1}\left(i \varrho_{1}\right) J_{1}\left(\varrho_{2}\right)\right]$. If we fix $\varrho_{1}$ and search for $\varrho_{2}$ (cf. figure 9), we notice that $\varrho_{2}$ possesses an infinity of discrete values which are nearly periodic. When $\varrho_{1}$ tends to nought, $\varrho_{2}$ tends to a root of $J_{1}, j_{1, k}$, where $k$ is an integer. When $\varrho_{1}$ tends to infinity, $\varrho_{2}$ tends to a root of $Y_{1}\left(\varrho_{2}\right), y_{1, k}$. As a result, $\varrho_{2}$ belongs to the successive intervals, $\left[y_{1,1}, j_{1,1}\right],\left[y_{1,2}, j_{1,2}\right], \ldots$ Inside each interval, $\varrho_{2}$ is determined uniquely. The graph $\left(\beta, c_{0}\right)$ is plotted in Figure 5 (b) for $U_{0}^{\prime}=1, \sigma=1$. The modes are shared between two branches. The upper one is bounded by the constraint $y \leq d$ at high $c_{0}$ and and the second by (L3) around $\beta \sim 2$. A third branch starting at $\beta \sim 2$ for small $c_{0}$ is forbidden due to the constraint (L3). When $\sigma=-1$, no modes are allowed, once again due to (L3). It is the same result as the Poiseuille flow but for opposite $\sigma$. Indeed, this time $U_{0}(y)$ and $U_{1}(y)$ are of opposite directions when $\sigma>0$. (L4) is always valided; so (L2) is except for very small values of $c_{0}$.

\section{2 order $\epsilon^{\frac{3}{2}}$}

The expressions of $\phi_{l}^{(1)}$ at either side of the critical level cannot be determined analytically due to the presence of the additional mean flow $U_{1}(y)$ in the right-hand side of (19) whose evolution is defined in terms of the error function. However, $\phi_{d}^{(1) \pm}$ are easily obtained through (19)

$$
\begin{aligned}
& \phi_{d}^{(1)+}=-\frac{2}{3}\left(\eta^{2}\left[\mathcal{A}^{+} J_{2}(\varrho)+\mathcal{B}^{+} Y_{2}(\varrho)\right]-\frac{\eta_{2}^{2} \mathcal{A}^{+}}{Y_{1}\left(\varrho_{2}\right)} \frac{\varrho}{\varrho_{2}}\left[Y_{1}(\varrho) J_{2}\left(\varrho_{2}\right)-Y_{2}\left(\varrho_{2}\right) J_{1}(\varrho)\right]\right)+\gamma_{d, 1}^{+} \phi^{+}, \\
& \phi_{d}^{(1)-}=\frac{2}{3} i\left(\frac{\eta^{2}}{3}\left[\mathcal{A}^{-} J_{2}(i \varrho)+\mathcal{B}^{-} Y_{2}(i \varrho)\right]-\frac{\eta_{1}^{2} \mathcal{A}^{-}}{Y_{1}\left(i \varrho_{1}\right)} \frac{\varrho}{\varrho_{1}}\left[Y_{1}(i \varrho) J_{2}\left(i \varrho_{1}\right)-Y_{2}\left(i \varrho_{1}\right) J_{1}(i \varrho)\right]\right)+\gamma_{d, 1}^{-} \phi^{-} .
\end{aligned}
$$

Hence, we deduce that, after expanding $\phi_{d}^{(1)}$ around $y_{c}$

$$
\gamma_{d, 1}^{+}=\beta_{d, 1}^{+}+\frac{2}{3} \frac{\eta_{2}^{2}}{\varrho_{2}} \frac{J_{2}\left(\varrho_{2}\right)}{J_{1}\left(\varrho_{2}\right)}, \quad \gamma_{d, 1}^{-}=\beta_{d, 1}^{-}+\frac{2}{3} \frac{\eta_{1}^{2}}{i \varrho_{1}} \frac{J_{2}\left(i \varrho_{1}\right)}{J_{1}\left(i \varrho_{1}\right)} .
$$


The Fredholm alternative for $\psi_{d}^{(1)}(52)$ yields

$$
\left[\alpha_{d, 1}\right]_{-}^{+}=\frac{1}{12 \bar{\beta}}\left(\frac{\varrho_{2}^{2}}{J_{1}^{2}\left(\varrho_{2}\right)}+\frac{\varrho_{1}^{2}}{J_{1}^{2}\left(i \varrho_{1}\right)}\right) .
$$

The $O\left(\epsilon^{2}\right)$ relations are given in the Appendix E.

\section{Conclusion}

In this study, we have explicitly analyzed the strong interaction of a free Rossby wave in a shear flow with a nonlinear critical layer in the long-wave régime. The presence of a flow in a nonlinear critical layer leads to a scaling which provides a stronger dispersion with shorter length scales and a shorter time scale than those which hold in the traditional KdV dynamics (Redekopp (1977)). For the flow within the separatrices in the nonlinear critical layer, we use a modified form of the Prandtl-Batchelor theorem. Our analysis, based on the hypothesis of a smooth and slow evolution of the flow within the nonlinear critical layer, leads to the derivation of a modified Korteweg-de-Vries equation at the third order of the asymptotic expansion in the inner flow. On the contrary, Brown and Stewartson (1979) investigated the more rapid evolution of long Rossby waves in the special case when $U_{0}^{\prime \prime}\left(y_{c}\right)=\beta$, which has the effect of removing the singularity at the leading order. They found that the dynamics was described by two coupled equations, derived at the second order, and which were not amenable to explicit integration. For instance, a nonlinear wave of wavenumber $O\left(\epsilon^{\frac{1}{4}}\right)$ in their approach evolves with the time $T=\epsilon^{\frac{3}{4}} t$, and experiences finite oscillations in vorticity at $T=O(1)$, which is damaging for the formation of solitary waves. For a smaller wavenumber $O\left(\epsilon^{\frac{1}{2}}\right)$, the $\mathrm{D}$-wave hypothesis was ruled out and the $\mathrm{E}$-wave motion also possessed such finite oscillations. Warn \& Warn (1978) obtained similar coupled temporal equations with $T=\epsilon^{\frac{1}{2}} t$ but for finite-period nonlinear waves. Their leading-order streamwise velocity had a jump through the critical layer and the computed critical-layer flow was very far from being solitary-like for small wavenumbers. Our slower time-scale allows us to find a zero velocity jump at the leading order as in previous steady critical layer studies (Benney and Bergeron (1969),Davis (1969)). Nevertheless, our higher-order analysis reveals a $O\left(\epsilon^{\frac{3}{2}}\right)$-velocity jump which causes a coupling of the outer and inner flows, which in turn modifies the KdV equation that emerges as a necessary relation in order that the $O\left(\epsilon^{\frac{3}{2}}\right)$ velocity may be matched all along the separatrices within the critical layer. The new nonlinear terms in the amplitude equation are the outcome of the interactions taking place in the critical layer and are not polynomials in the amplitude. Such an equation would seem not to be integrable. However, solitary wave solutions exist and are found explicitly. We also note that Hickernell (1984) derived a temporal evolution equation for a weakly nonlinear Rossby wave critical layer on a hyperbolic tangent basic velocity profile. Its very rapid time scaling of $\epsilon^{-\frac{2}{5}}$ yielded a nonlinear term result in the form of a convolution integral, with the consequence that the amplitude equation was not integrable.

We have found that the flow within the separatrices must obey certain topological conditions, which are unlikely to be satisfied after matching the outer flow (outside the critical layer) with the inner flow (within the critical layer) unless the streamline geometry within the critical layer is adjusted by the introduction of a strained coordinate. However, this parametrization is still insufficient to describe the critical-layer flow around the dividing streamlines in the case of the E-wave (see section 4.1 and Figure 1). A second additional layer with its own scaling and variable must then be introduced to cancel a certain singularity. This additional layer is not specific to this problem, but appears whenever one wishes to accurately model the motion in the neighbourhood of the separatrices without using viscosity, and instead by only taking into account of nonlinearity. For instance, such an additional layer was used by Caillol and Grimshaw (2004) to describe the strong critical-layer interaction between an aximmetric vortex and an azimuthal mode. Due to the technical complexity of the E-wave modelling, we have postponed its study for a subsequent article.

Our analysis of the existence of singular neutral modes needs the simultaneous study of all flows: undisturbed, diffusive and critical-layer flows. We have found that an infinity of modes exist, but only for certain $\beta$-domains. The condition relating to the compatibility of the amplitude equation vis-à-vis the solitary wave solutions, is not as stringent as the condition for the existence of the diffusive bounday layers with respect to the choices of the undisturbed mean flows: $U_{0}(y)$ and $U_{u, 1}(y)$. Concerning the physical relevance of these solutions, the main issue is whether the present long-time régime, evolving with the slow time $T=\epsilon^{\frac{5}{4}}$ is possible in practice, and in particular whether the free Rossby wave mode 
does not decay before the nonlinear dynamics within the critical layer can be established. One approach to examine this issue would be to consider the linear initial-value problem, in order to see if Rossby waves with $O\left(\epsilon^{\frac{1}{4}}\right)$ wavenumbers can survive the algebraic decay of free modes. Another approach would be to consider the stability of the solitary waves found here, probably through direct numerical simulations. In this context, we note that Maslowe and Clarke (2002) found some evidence for the existence of Rossby waves in the presence of a nonlinear critical layer in a numerical study of resonant wave interactions.

\section{References}

Batchelor, G. (1956). On steady laminar flow with closed streamlines at large Reynolds number. J. Fluid Mech., 1:177-190.

Batchelor, G. (1967). Flow of a uniform incompressible viscous fluid. In An introduction to fluid dynamics, pages 174-263. edited by Cambridge University Press, Cambridge.

Benney, D. J. (1966). Long nonlinear waves in fluid flows. Journ. Math. Phy., 45:42-63.

Benney, D. J. and Bergeron, R. F. (1969). A new class of nonlinear waves in parallel flows. Studies in Applied Maths., 48:181-204.

Bradshaw, N. G., Vaughan, G., and Ancellet, G. (2002). Generation of layering in the lower stratosphere by a breaking Rossby wave. J. Geophys. Res. Atm., 107(D1-D2).

Brown, S. N. and Stewartson, K. (1978). The evolution of the critical layer of a Rossby wave. part ii. Geophys. Astrophys. Fluid Dyn., 10:1-24.

Brown, S. N. and Stewartson, K. (1979). On the secular stability of a regular Rossby neutral mode. Geophys. Astrophys. Fluid Dyn., 14:1-18.

Brunet, G. and Warn, T. (1990). Rossby wave critical layers on a jet. J. Atmos. Sci., 47(10):1173-1178.

Caillol, P. and Grimshaw, R. H. (2004). Steady multi-polar planar vortices with nonlinear critical layers. Geophys. Astrophys. Fluid Dyn., 98:473-506.

Davis, R. E. (1969). On the high Reynolds number flow over a wavy boundary. J. Fluid Mech., 36:337-346.

Fritts, D. C. (1978). The nonlinear gravity wave-critical level interaction. J. Atmos. Sci., 35:397.

Haberman, R. (1972). Critical layers in parallel flows. Studies in Applied Maths., L1(2):139-161.

Haynes, P. (1985). Nonlinear instability of a Rossby-wave critical layer. J. Fluid Mech., 161:493-511.

Haynes, P. (1989). The effect of barotropic instability on the nonlinear evolution of a Rossby-wave critical level. J. Fluid Mech., 207:231-266.

Hickernell, F. J. (1984). Time-dependent critical layers in shear flows on the beta-plane. J. Fluid Mech., 142:431-449.

Kivshar, Y. S. and Malomed, B. A. (1989). Dynamics of solitons in nearly integrable systems. Rev. of Mod. Phys., 61(4):763-916.

Kizner, Z. (1997). Solitary Rossby waves with baroclinic modes. J. Marine Res., 55:671-685.

Long, R. (1964). Solitary waves in the westerlies. J. Atmos. Sci., 21:197-200.

Luo, D. (1996). Envelope solitary Rossby waves and modulational instabilities of uniform Rossby wave trains in two space dimensions. Wave Motion, 24:315-325.

Malguzzi, P. and Malanote-Rizzoli, P. (1985). Barotropic modon propagation over slowly varing topography. Geophys. Astrophys. Fluid Dyn., 42:2463-2477.

Maslowe, S. A. and Clarke, S. R. (2002). Subcritical Rossby waves in zone shear flows with nonlinear critical layers. Studies in Applied Maths., 108:89-103. 
Maxworthy, T. and Redekopp, L. G. (1976). A solitary wave theory of the Great Red Spot and other observed features in the Jovian atmosphere. Icarus, 29:261-271.

McIntyre, M. E. and Palmer, T. N. (1985). A note on the general concept of wave breaking for Rossby and gravity waves. Pure Appl. Geophys., 123:964-975.

Pelinovskiy, D. and Grimshaw, R. (1996). An asymptotic approach to solitary wave instability and critical collapse in long-wave KdV-type evolution equations. Physica D, 98:139-155.

Redekopp, L. G. (1977). On the theory of solitary Rossby waves. J. Fluid Mech., 82(4):725-745.

Rhines, P. B. and Young, W. R. (1982). Homogenization of potential vorticity in planetary gyres. J. Fluid Mech., 122:347-367.

Stewartson, K. (1981). Marginally stable inviscid flows with critical layers. IMA, Journ. of Applied Maths, 27:133-175.

Swaters, G. E. (1986). Barotropic modon propagation over slowly varing topography. Geophys. Astrophys. Fluid Dyn., 36:85-113.

Troitskaya, Y. and Reznik, S. (1996). Quasi-steady dissipative nonlinear critical layer in a stratified shear flow. Phys. of Fluids, 8(12):3313-3328.

Vanneste, J. (1998). A nonlinear critical layer generated by the interaction of free Rossby waves. J. Fluid Mech., 371:319-344.

Wang, L., Koblinsky, C., Howden, S., and B., B. (1998). Large-scale Rossby wave in the mid-latitude South Pacific from altimetry data. Geophys. Res. Lett., 25(2):179-182.

Warn, T. and Warn, H. (1978). The evolution of a nonlinear critical level. Studies in Applied Maths., 59:37-71. 


\section{Appendices}

\section{A The outer flow}

\section{A1 First terms in Frobenius series}

A1.1 $O(\epsilon)$

$$
b_{0,3}=\frac{1}{72}\left[6 \bar{U}_{0}^{I V}-5 b_{0}^{3}-4 b_{0}^{2} \bar{U}_{0}^{\prime \prime}-12 b_{0} \bar{U}_{0}^{\prime \prime \prime}-6 \bar{\beta} \bar{U}_{0}^{\prime \prime \prime}+3 \bar{\beta} \bar{b}_{0}\left(4 U_{0}^{\prime \prime}-3 \beta\right)\right]
$$

A1.2 $O\left(\epsilon^{\frac{3}{2}}\right)$

$$
\begin{gathered}
c_{l, 1,0}=\bar{U}_{1}^{\prime}-\frac{\bar{U}_{1}^{\prime \prime}}{b_{0}}, c_{l, 1,1}=0 \\
b_{l, 1,0}=0, b_{l, 1,1}=0, b_{l, 1,2}=\bar{b}_{0}\left(U_{1}^{\prime \prime}-b_{0} U_{1}^{\prime}\right) / 2 \\
c_{d, 1,2}=-1 / 2 \\
b_{v, 1,0}=0, b_{v, 1,1}=\bar{U}_{0}^{\prime \prime \prime}, b_{v, 1,2}=\frac{1}{2} \bar{b}_{0} U_{0}^{\prime \prime \prime}, \\
c_{v, 1,0}=0, c_{v, 1,1}=0, c_{v, 1,2}=\left(2 \bar{U}_{0}^{I V}-3 \bar{b}_{0} U_{0}^{\prime \prime \prime}-\bar{U}_{0}^{\prime \prime} \bar{U}_{0}^{\prime \prime \prime}\right) / 4
\end{gathered}
$$

A1.3 $O\left(\epsilon^{2}\right)$

$$
\begin{aligned}
& b_{l, 2,0}=-\bar{b}_{0} V_{0}, b_{l, 2,1}=0, \\
& c_{l, 2,0}=\frac{1}{2}\left\{\bar{T}_{0} \bar{U}_{0}^{\prime \prime}-b_{0}^{-1}\left[\left(2 a b_{0}^{2}+3 b_{0}^{2}+4 b_{0,2}\right) \bar{T}_{0}+2\left(c_{l, 1,0}+\beta_{l, 1}\right) \bar{U}_{1}^{\prime \prime}+2\left(\bar{U}_{2}^{\prime \prime}-\bar{b}_{0} U_{2}^{\prime}-\beta_{l, 1} \bar{b}_{0} U_{1}^{\prime}\right)\right]\right\}, \\
& a_{n, 2,0}=b_{0} \bar{b}_{0}, a_{n, 2,1}=\bar{b}_{0}\left(3 a_{0,3}-b_{0,2}+\frac{1}{4} \beta \bar{b}_{0}\right), \\
& b_{n, 2,0}=2 \bar{b}_{0} b_{0}\left(1+a-\frac{1}{4} \hat{\beta}\right)-\bar{b}_{0} R_{0}, b_{n, 2,1}=0, \\
& c_{n, 2,0}=-\frac{1}{2} \bar{b}_{0}, \\
& b_{d, 2,0}=-\bar{b}_{0} S_{0}, b_{d, 2,1}=0, \\
& c_{d, 2,0}=-\bar{S}_{0}\left(a b_{0}-\bar{U}_{0}^{\prime \prime}+\frac{U_{0}^{\prime \prime \prime}}{b_{0}}\right)+\beta_{d, 1}\left(\bar{U}_{1}^{\prime}-\frac{\bar{U}_{1}^{\prime \prime}}{b_{0}}\right), c_{d, 2,1}=0, \\
& b_{c, 2,0}=\bar{b}_{0}, b_{c, 2,1}=0 \text {, } \\
& c_{c, 2,0}=\frac{1}{2}\left(2 a \bar{b}_{0}+3 \bar{b}_{0}+4 \frac{\bar{b}_{0,2}}{b_{0}}-\overline{\bar{U}}_{0}^{\prime \prime}\right) \text {, } \\
& c_{n, 2,1}=\frac{6}{\beta-U_{0}^{\prime \prime}}\left[2 b_{0}^{3}\left(1-a^{2}\right)+2 a_{0,3} b_{0}(6 a+5)-6 b_{0} b_{0,2}\right. \\
& \left.+12 b_{0,3}+b_{0}^{2} R_{0}+4 b_{0,2} R_{0}-2 a b_{0}\left(b_{0}^{2}+2 b_{0,2}-b_{0} R_{0}\right)\right] \\
& +\frac{1}{12}\left(2\left\{3\left(2 a \bar{b}_{0}+6 \bar{b}_{0}-\bar{R}_{0}\right) \bar{U}_{0}^{\prime \prime}-2\left[\bar{\beta}\left(6 a \bar{b}_{0}+6 \bar{b}_{0}-3 \bar{R}_{0}\right)-\bar{C}_{0}^{\prime \prime \prime}\right]\right\}-3 \overline{\bar{\beta}}\left(\bar{U}_{0}^{\prime \prime}-2 \bar{\beta}\right)\right), \\
& c_{c, 2,1}=0, b_{f, 2,0}=-\bar{b}_{0} D_{0}, b_{f, 2,1}=0, \\
& c_{f, 2,0}=0, c_{f, 2,1}=\beta_{d, 1} / b_{0}, \quad\left(D_{0}=0\right) .
\end{aligned}
$$




\section{A2 Outer expansion expressed with the inner variable $Y$}

The expansion of the streamfunction around the critical-layer edges is

$$
\begin{aligned}
\lim _{Y \rightarrow s \infty} \Psi= & \epsilon\left\{\frac{1}{2} U_{0}^{\prime} Y^{2}+A^{*}+\epsilon^{\frac{1}{2}} \ln \epsilon \frac{1}{2} b_{0} Y A^{*}\right. \\
+\epsilon^{\frac{1}{2}}\left[\frac{1}{6}\right. & \left.U_{0}^{\prime \prime} Y^{3}+\frac{1}{2} U_{1}^{\prime s} Y^{2}+\left[c_{l, 1,0}^{s}+\beta_{l, 1}^{s}+b_{0}\left(a^{s}+\ln \left|Y^{*}\right|\right) Y\right] A^{*}+\beta_{d, 1} \partial_{X}^{2} A^{*}+c_{n, 2,0} \frac{A^{* 2}}{2 Y}\right] \\
& +\epsilon \ln ^{2} \epsilon \frac{1}{8} b_{0} \bar{b}_{0} A^{* 2}+\epsilon \ln \epsilon \frac{1}{2}\left[\left(b_{l, 2,0}+\beta_{l, 1}^{s} b_{0} Y+\frac{1}{2} b_{0}^{2} Y^{2}\right) A^{*}+\left(\frac{1}{2} b_{n, 2,0}+b_{0} \bar{b}_{0} \ln \left|Y^{*}\right|\right) A^{* 2}\right. \\
& \left.+\left(b_{d, 2,0}+b_{0} \beta_{d, 1} Y\right) \partial_{X}^{2} A^{*}+b_{f, 2,0} \partial_{X}^{4} A^{*}+U_{0}^{\prime} b_{c, 2,0} \mathcal{C}[A]\right] \\
+\epsilon\left[U_{0}^{\prime \prime \prime} \frac{Y^{4}}{4 !}+U_{1}^{\prime \prime} s \frac{Y^{3}}{6}+U_{2}^{\prime} \frac{Y^{2}}{2}\right. & \\
+ & {\left[c_{l, 2,0}^{s}+\beta_{l, 2}^{s}+b_{l, 2,0} \ln Y^{*}+\left(\alpha_{l, 1}^{s}+\beta_{l, 1}^{s} b_{0} \ln \left|Y^{*}\right|\right) Y+\left(b_{0,2}+\frac{1}{2} b_{0}^{2} a_{l, 1}^{s}+\frac{1}{2} b_{0}^{2} \ln \left|Y^{*}\right|\right) Y^{2}\right] A^{*} } \\
& +\frac{1}{2}\left[c_{n, 2,1}^{s}+b_{n, 2,0} \ln \left|Y^{*}\right|+a_{n, 2,0} \ln { }^{2}\left|Y^{*}\right|+\frac{1}{2} \frac{c_{n, 4,0}^{s}}{Y}\right] A^{* 2} \\
+ & {\left[c_{d, 2,0}^{s}+\beta_{d, 2}+b_{d, 2,0} \ln \left|Y^{*}\right|+\left(\alpha_{d, 1}^{s}+b_{0} \beta_{d, 1} \ln \left|Y^{*}\right|\right) Y\right] \partial_{X}^{2} A^{*}+\left(c_{f, 2,0}+\beta_{f, 2}+b_{f, 2,0} \ln \left|Y^{*}\right|\right) \partial_{X}^{4} A^{*} } \\
+ & \left.\left.U_{0}^{\prime}\left(c_{c, 2,0}+\beta_{c, 2}+b_{c, 2,0} \ln Y^{*}\right) \mathcal{C}[A]\right]+O\left(\epsilon^{\frac{3}{2}} \ln { }^{2} \epsilon, \epsilon^{\frac{3}{2}} \ln \epsilon, \epsilon^{\frac{3}{2}}\right)\right\}
\end{aligned}
$$

\section{B Extended Prandtl-Batchelor Theorem}

The momentum equation underlying the vorticity equation (3) is within the separatrices

$$
\partial_{t} \mathbf{u}+\mathbf{u} \times \mathbf{Q}+\nabla H=\lambda \epsilon^{\frac{7}{4}} \nabla^{2} \mathbf{u}+\lambda \epsilon^{\frac{7}{4}} \mathbf{F},
$$

where $H=p / \rho+\frac{1}{2}|\mathbf{u}|^{2}, \mathbf{u}=\left(\Psi_{y},-\Psi_{x}, 0\right)$ and $\mathbf{Q}=Q_{\text {total }} \hat{\mathbf{z}}$. The fluid variables will again be decomposed into inviscid and viscous parts. Because the time-scale is slow (see 8), we shall assume here for simplicity, that the flow is stationary in the reference frame moving with the wave, since our main concern is with steady waves. Also, the time evolution term does not affect the lowest-order vorticity terms.

We now adapt the arguments of Batchelor (1956) and Rhines and Young (1982) to the present situation, where it is necessary inter alia to take account of the body force term. We study an imaginary closed-streamline flow that can be constructed by cutting the $\mathrm{D}$-wave flow on the axis $\zeta=0$ (see figure 1 (a)) and swapping the left and right parts so that the right part be at the left and the resulting flow look like a E-wave motion. Consider then a family of closed streamlines, and integrate B2 around a streamline to get

$$
\oint \mathbf{u} \times \mathbf{Q} \cdot d \mathbf{l}+\oint \nabla H \cdot d \mathbf{l}+\lambda \epsilon^{\frac{7}{4}} \oint(\nabla \times \mathbf{Q}) \cdot d \mathbf{l}-\lambda \epsilon^{\frac{7}{4}} \oint U_{0}^{\prime \prime}(y) \mathbf{x} \cdot d \mathbf{l}+\epsilon^{\frac{5}{4}} \oint \partial_{T} \mathbf{u} \cdot d \mathbf{l}=0,
$$

where $d \mathbf{l}$ is the line element. The two first terms (both inviscid and viscous) vanish. Further for a steady travelling wave $\partial_{T} \mathbf{u}=-V \partial_{X} \mathbf{u}$, the last inviscid integral vanishes by symmetry. Within the critical layer, $\mathbf{u}$ is $O(\epsilon)$ relative to $\nabla \times \mathbf{Q}$, so the last viscous term can be ignored to leading orders. (B3) then reduces to

$$
\oint\left(\nabla \times \mathbf{Q}-U_{0}^{\prime \prime}(y) \hat{\mathbf{x}}\right) \cdot d \mathbf{l}=0 .
$$

We now expand the potential vorticity,

$$
Q_{\text {total }}=\frac{1}{\epsilon} \partial_{Y}^{2} \Psi+\epsilon^{\frac{1}{2}} \partial_{X}^{2} \Psi-f_{0}-\epsilon^{\frac{1}{2}} \beta Y=Q_{0}+\epsilon^{\frac{1}{2}} Q_{2}+\epsilon \ln \epsilon Q_{4}+\epsilon Q_{5}+\cdots
$$

As in Batchelor (1956) and Rhines and Young (1982), we introduce new coordinates along and perpendicular to the closed streamline. This converts (B4) to

$$
\oint|\mathbf{u}| \frac{\partial Q_{t o t a l}}{\partial \Psi} d l-\oint_{30} U_{0}^{\prime \prime}(y) \hat{\mathbf{x}} \cdot d \mathbf{l}=0 .
$$


It is now readily established that at the leading order,

$$
Q_{0}=\text { constant }=U_{0}^{\prime}-f_{0},
$$

while at the next order

$$
Q_{2}=\text { constant }=\frac{1}{2}\left(U_{1}^{\prime+}+U_{1}^{\prime-}\right) .
$$

Here, the evaluation of the constant comes from the matching with the flow outside the separatrices. At the next order also

$$
Q_{4}=\text { constant }
$$

However, at the order $O(\epsilon)$ we find that

$$
Q_{5}=U_{0}^{\prime \prime \prime} S+\text { constant }
$$

Continuing this process, we eventually find that $Q_{8}$ is not constant within the region of closed streamlines.

\section{New streamline definition}

The new inner flow when expressed with the variable $\tilde{S}$ is displayed until the order $\epsilon^{2} \ln \epsilon$ for the vorticity, streamwise velocity and streamfunction.

\section{C1 order $\epsilon^{\frac{3}{2}}$}

$$
\begin{gathered}
\tilde{Q}^{(2)}=Q, \quad \tilde{Q}^{(2, c)}=Q_{2}, \quad \tilde{\Psi}_{Y Y}^{(2)}=\Psi_{Y Y}^{(2)}, \\
\tilde{\Psi}_{Y}^{(2)}=b_{0} \mathcal{G}(A, \tilde{S})+\beta(\tilde{S}-A)+U_{1}^{\prime} \tilde{S}_{\tilde{Y}}+\mathcal{U}^{(2)}-\tilde{\mathcal{U}}_{2}, \quad \tilde{\Psi}_{Y}^{(2, c)}=Q_{2} \tilde{S}_{\tilde{Y}}+\beta(\tilde{S}-A),
\end{gathered}
$$

with $\tilde{\mathcal{U}}_{2}=b_{0} \mathcal{G}\left(A, \tilde{S}_{c}\right)+\frac{1}{2} \tilde{Y}_{s}\left[U_{1}^{\prime}\right]_{-}^{+}+\mathcal{U}^{(2)}$.

$$
\begin{gathered}
\tilde{\Psi}^{(2)}=\Psi^{(2)}(\tilde{S})+U_{0}^{\prime} \varphi^{(2)}(\tilde{S}) \\
\text { order } \epsilon^{2} \ln ^{2} \epsilon
\end{gathered}
$$

\section{C1.1 order $\epsilon^{2} \ln \epsilon$}

The vorticity distortion has not only vanished but vorticity is equal in open and closed-streamline flows:

$$
\tilde{Q}^{(4)}=\tilde{Q}^{(4, c)}=0, \quad \tilde{\Psi}_{Y Y}^{(4)}=\tilde{\Psi}_{Y Y}^{(4, c)}=-\frac{1}{2} \beta b_{0} A .
$$

Both velocities and streamfunctions are equal as well:

$$
\begin{gathered}
\tilde{\Psi}_{Y}^{(4)}=-\frac{1}{2} \beta b_{0} \tilde{S}_{\tilde{Y}} A, \quad \tilde{\Psi}_{Y}^{(4, c)}=-\frac{1}{2} \beta b_{0} \tilde{S}_{\tilde{Y}} A-\mathcal{U}^{(4)}-\frac{1}{2} b_{0} Q_{2} A+\tilde{\mathcal{U}}_{4}, \\
\tilde{\Psi}^{(4)}=-\frac{1}{2} b_{0} A\left(\mathcal{U}^{(2)}+\beta \tilde{S}\right)+\Phi^{(4)}, \quad \tilde{\Psi}^{(4, c)}=-\frac{1}{2} b_{0} A\left(\tilde{\mathcal{U}}_{2}+\beta \tilde{S}\right)+\tilde{\Phi}_{4},
\end{gathered}
$$

with $\tilde{\mathcal{U}}_{4}=\mathcal{U}^{(4)}+\frac{1}{2} b_{0} Q_{2} A$ and $\tilde{\Phi}_{4}=\frac{1}{2} b_{0}\left(\tilde{\mathcal{U}}_{2}-\mathcal{U}^{(2)}\right) A+\Phi^{(4)}$.

\section{Diffusive boundary layer flow}

The vorticity and $\kappa$ are expanded following $\epsilon^{\frac{1}{2}} / \nu$

$$
Q_{D B}^{(2)}(H, \nu)=\sum_{k=0}\left(\frac{\epsilon^{\frac{1}{2}}}{\nu}\right)^{k} Q_{k}^{(2)}(H), \quad \kappa=\sum_{k=0}\left(\frac{\epsilon^{\frac{1}{2}}}{\nu}\right)^{k} \kappa_{k} .
$$

The related vorticity jump was determined in the critical layer study (see 33) and is

$$
\left[U_{1}^{\prime}\right]_{-}^{+}=-2\left[U_{0}^{\prime \prime}(y c)-\beta\right] Y_{\infty}=Q_{D B}^{(2)}\left(0^{+}, \nu\right)-Q_{D B}^{(2)}\left(0^{-}, \nu\right),
$$


the vorticity gradient also possesses a jump (see 39)

$$
\left[U_{1}^{\prime \prime}\right]_{-}^{+}=-6\left[U_{0}^{\prime \prime}(y c)-\beta\right]^{2} \frac{Y_{\infty}}{U_{0}^{\prime}}=Q_{D B, H}^{(2)}\left(0^{+}, \nu\right)-Q_{D B, H}^{(2)}\left(0^{-}, \nu\right) .
$$

Let us assume that the above jumps are also expanded like in (D5). Each $Q_{k}^{(2)}$ then satisfies

$$
Q_{k, H H}^{(2)}-2 k Q_{k}^{(2)}+2 H Q_{k, H}^{(2)}+2 \sum_{j=0}^{k}(1+j) \kappa_{j} Q_{k-j, H}^{(2)}=0
$$

The boundary conditions at infinity are based upon the existence of an undisturbed mean flow (denoted by the subscript $u$ ) that must be nevertheless perturbed vis-à-vis the basic flow $U_{0}$ in the form

$$
U_{u}(y)=U_{0}(y)+\epsilon^{\frac{1}{2}} U_{u, 1}(y)+\epsilon U_{u, 2}(y)+\ldots
$$

$U_{u}$ is expanded around $y_{c 0}$. Dropping $U_{0}$ that is useless and letting $H$ appear through $y$, we have when $H \rightarrow \infty$

$Q_{D B, H}^{(2)} \rightarrow \frac{\epsilon^{\frac{1}{2}}}{\nu} U_{u, 1}^{\prime \prime}\left(y_{c 0}\right), Q_{D B}^{(2)} \rightarrow U_{u, 1}^{\prime}\left(y_{c 0}\right)+\frac{\epsilon^{\frac{1}{2}}}{\nu} U_{u, 1}^{\prime \prime}\left(y_{c 0}\right)(H+\kappa), U_{D B}^{(2)} \rightarrow U_{u, 1}^{\prime}\left(y_{c 0}\right)(H+\kappa)+\frac{\epsilon^{\frac{1}{2}}}{\nu} \frac{1}{2} U_{u, 1}^{\prime \prime}\left(y_{c 0}\right)(H+\kappa)^{2}$.

The boundary conditions on the edges of the critical layer are derived by using the mean flow expansion around $y_{c}$ of the first section expressed through the DB inner variable $H$, so when $H \rightarrow 0$

$$
Q_{D B, H}^{(2)} \rightarrow \frac{\epsilon^{\frac{1}{2}}}{\nu} U_{1}^{\prime \prime}\left(y_{c}\right), \quad Q_{D B}^{(2)} \rightarrow U_{1}^{\prime}\left(y_{c}\right)+\frac{\epsilon^{\frac{1}{2}}}{\nu} U_{1}^{\prime \prime}\left(y_{c}\right) H, \quad U_{D B}^{(2)} \rightarrow U_{1}^{\prime}\left(y_{c}\right) H+\frac{\epsilon^{\frac{1}{2}}}{\nu}\left[\frac{1}{2} U_{1}^{\prime \prime}\left(y_{c}\right) H^{2}+u_{10}\right] .
$$

We will prove the presence of the small velocity $u_{10}$ later on. The expansion of the distorted flow $U_{1}$ around the critical layer is therefore

$$
U_{1}=U_{1}\left(y_{c}\right)+\frac{\epsilon}{\nu^{2}} u_{10}+U_{1}^{\prime}\left(y-y_{c}\right)+\frac{1}{2} U_{1}^{\prime \prime}\left(y-y_{c}\right)^{2}+\ldots
$$

$u_{10}$ is absorbed in the expansion of $c$.

We solve the equation D6 to the two first orders. The leading-order vorticity $Q_{0}^{(2)}$ is given by

$$
Q_{0}^{(2)}=U_{u, 1}^{\prime}+q_{10}^{s} \int_{s \infty}^{H+\kappa} e^{-u^{2}} d u
$$

It is the classical error function evolution of a laminar boundary layer (Batchelor (1967)). The boundary conditions on the critical-layer edges yield

$$
q_{10}^{+} \int_{+\infty}^{\kappa_{0}} e^{-u^{2}} d u-q_{10}^{-} \int_{-\infty}^{\kappa_{0}} e^{-u^{2}} d u=\left[U_{1,0}^{\prime}\right]_{-}^{+}
$$

The leading-order streamwise velocity being

$$
U_{0}^{(2)}=U_{u, 1}^{\prime}\left(H+\kappa_{0}\right)+q_{10}^{s}\left(\left(H+\kappa_{0}\right) \int_{s \infty}^{H+\kappa_{0}} e^{-u^{2}} d u+\frac{1}{2} e^{-\left(H+\kappa_{0}\right)^{2}}\right)
$$

we have two other boundary conditions for the velocity

$$
q_{10}^{-}\left[e^{-\kappa_{0}^{2}}+2 \kappa_{0}(\sqrt{\pi}-J)\right]=q_{10}^{+}\left[e^{-\kappa_{0}^{2}}-2 \kappa_{0} J\right]=-2 \kappa_{0} U_{u, 1}^{\prime},
$$

with $J=\int_{\kappa_{0}}^{\infty} e^{-u^{2}} d u$. Finally, $q_{10}^{s}$ are

$$
q_{10}^{+}=-\frac{2 \kappa_{0} U_{u, 1}^{\prime}}{e^{-\kappa_{0}^{2}}-2 \kappa_{0} J}, \quad q_{10}^{-}=-\frac{2 \kappa_{0} U_{u, 1}^{\prime}}{e^{-\kappa_{0}^{2}}+2 \kappa_{0}(\sqrt{\pi}-J)}
$$

$\kappa_{0}$ satisfies

$$
2 \kappa_{0} \sqrt{\pi} U_{u, 1}^{\prime}=\left[U_{1,0}^{\prime}\right]_{-}^{+} e^{\kappa_{0}^{2}}\left[e^{-\kappa_{0}^{2}}+2 \kappa_{0}(\sqrt{\pi}-J)\right]\left[e^{-\kappa_{0}^{2}}-2 \kappa_{0} J\right] .
$$

This relation admits a unique and finite solution provided $U_{u, 1}^{\prime}$ is non-zero. In this case, $\kappa_{0}$ is an odd function of $U_{u, 1}^{\prime}$. 


\section{D1 order $\epsilon^{\frac{1}{2}} / \nu$}

The first-order vorticity $Q_{1}^{(2)}$ satisfies the equation

$$
Q_{1, H H}^{(2)}-2 Q_{1}^{(2)}+2\left(H+\kappa_{0}\right) Q_{1, H}^{(2)}=-4 \kappa_{1} Q_{0, H}^{(2)} ;
$$

its general solution having the right behaviour at $H \rightarrow \infty$ is

$$
Q_{1}^{(2)}=U_{u, 1}^{\prime \prime}\left(H+\kappa_{0}\right)+q_{11}^{s}\left(\left(H+\kappa_{0}\right) \int_{s \infty}^{H+\kappa_{0}} e^{-u^{2}} d u+\frac{1}{2} e^{-\left(H+\kappa_{0}\right)^{2}}\right)+\kappa_{1} q_{10}^{s} e^{-\left(H+\kappa_{0}\right)^{2}} .
$$

The related velocity is obtained after integration over $H$

$U_{1}^{(2)}=\frac{1}{2} U_{u, 1}^{\prime \prime}\left(H+\kappa_{0}\right)^{2}+\frac{1}{4} q_{11}^{s}\left(\left[1+2\left(H+\kappa_{0}\right)^{2}\right] \int_{s \infty}^{H+\kappa_{0}} e^{-u^{2}} d u+\left(H+\kappa_{0}\right) e^{-\left(H+\kappa_{0}\right)^{2}}\right)+\kappa_{1} q_{10}^{s} \int_{s \infty}^{H+\kappa_{0}} e^{-u^{2}} d u$.

The integration constants $q_{11}^{s}, \kappa_{1}$ and $u_{10}$ are determined by the following relations. The vorticity gradient jump gives

$$
q_{11}^{+} J+q_{11}^{-}(\sqrt{\pi}-J)+2 \kappa_{1}\left[q_{10}\right]_{-}^{+} \kappa_{0} e^{-\kappa_{0}^{2}}=-\left[U_{1,0}^{\prime \prime}\right]_{-}^{+},
$$

as for the vorticity jump

$$
q_{11}^{+}\left[\frac{1}{2} e^{-\kappa_{0}^{2}}-\kappa_{0} J\right]-q_{11}^{-}\left[\kappa_{0}(\sqrt{\pi}-J)+\frac{1}{2} e^{-\kappa_{0}^{2}}\right]+\kappa_{1}\left[q_{10}\right]_{-}^{+} e^{-\kappa_{0}^{2}}=\left[U_{1,1}^{\prime}\right]_{-}^{+},
$$

whereas the matching of the streamwise velocity on the critical-layer edges yields

$q_{11}^{+}\left(\kappa_{0} e^{-\kappa_{0}^{2}}-\left(1+2 \kappa_{0}^{2}\right) J\right)-4 \kappa_{1} q_{10}^{+} J=q_{11}^{-}\left(\left(1+2 \kappa_{0}^{2}\right)(\sqrt{\pi}-J)+\kappa_{0} e^{-\kappa_{0}^{2}}\right)+4 \kappa_{1} q_{10}^{-}(\sqrt{\pi}-J)=-2 \kappa_{0}^{2} U_{u, 1}^{\prime \prime}+4 u_{10}$.

$u_{10}$ can be chosen distortionless. We express $\kappa_{1}$ with the help of D10 and injects it in D11 and D12, $q_{11}^{s}$ are then univoquely determined, then $\kappa_{1}$ is obtained through D10. Finally, $\kappa_{1}$ and $q_{11}^{s}$ are

$$
\begin{gathered}
\kappa_{1}=-\frac{\left(2 \kappa_{0}\left[U_{1,1}^{\prime}\right]_{-}^{+}+\left[U_{1,0}^{\prime \prime}\right]_{-}^{+}\right)}{4\left[U_{1,0}^{\prime}\right]_{-}^{+}}, \\
q_{11}^{+}=-\frac{e^{\kappa_{0}^{2}}}{\sqrt{\pi}}\left(\left\{\left(1+\kappa_{0}^{2}\right) D^{-}+\kappa_{0}(\sqrt{\pi}-J)\right\}\left[U_{1,0}^{\prime \prime}\right]_{-}^{+}+2\left\{(\sqrt{\pi}-J)\left(\kappa_{0}^{2}-1\right)+\kappa_{0}^{3} D^{-}\right\}\left[U_{1,1}^{\prime}\right]_{-}^{+}\right), \\
\left.q_{11}^{-}=-\frac{e^{\kappa_{0}^{2}}}{\sqrt{\pi} \kappa_{0}}\left(\left\{\left(1+\kappa_{0}^{2}\right)\left[\kappa_{0} D^{+}-J\right]+J\right\}\left[U_{1,0}^{\prime \prime}\right]_{-}^{+}+2 \kappa_{0}\left\{\kappa_{0}^{2}\left[\kappa_{0} D^{+}-J\right]+J\right\}\left[U_{1,1}^{\prime}\right]_{-}^{+}\right\}\right) .
\end{gathered}
$$

$u_{10}$ is constrained by the relation

$$
\begin{aligned}
u_{10}=\frac{1}{2} \kappa_{0}^{2} U_{u, 1}^{\prime \prime}-\frac{\kappa_{0} e^{\kappa_{0}^{2}}}{4 \sqrt{\pi}}\{[ & \left.\left.\kappa_{0} D^{+}-J\right]\left[\kappa_{0} D^{-}+\sqrt{\pi}-J\right]+D^{+} D^{-}\right\}\left[U_{1,0}^{\prime \prime}\right]_{-}^{+} \\
& -\frac{e^{\kappa_{0}^{2}}}{2 \sqrt{\pi}}\left\{\left(\kappa_{0}^{2}-1\right)\left[\kappa_{0} D^{+}-J\right]\left[\kappa_{0} D^{-}+\sqrt{\pi}-J\right]+\kappa_{0}^{2} D^{+} D^{-}\right\}\left[U_{1,1}^{\prime}\right]_{-}^{+},
\end{aligned}
$$

with $D^{+}=e^{-\kappa_{0}^{2}}-2 \kappa_{0} J, D^{-}=e^{-\kappa_{0}^{2}}+2 \kappa_{0}(\sqrt{\pi}-J)$.

The first-order vorticity within the cat's eye is thus

$$
\begin{gathered}
Q_{2}=U_{u, 1}^{\prime}+\left[2 J-\sqrt{\pi}+4 \kappa_{0} J(\sqrt{\pi}-J) e^{\kappa_{0}^{2}}\right] \frac{\left[U_{1,0}^{\prime}\right]_{-}^{+}}{2 \sqrt{\pi}}+\frac{\epsilon^{\frac{1}{2}}}{\nu}\left[\kappa_{0} U_{u, 1}^{\prime \prime}\right. \\
-\frac{e^{\kappa_{0}^{2}}}{4 \sqrt{\pi}}\left(\left\{\left(1+\kappa_{0}^{2}\right) D^{-}+\kappa_{0}(\sqrt{\pi}-J)\right\} D^{+}+\left\{\left(1+\kappa_{0}^{2}\right)\left[\kappa_{0} D^{+}-J\right]+J\right\} \frac{D^{-}}{\kappa_{0}}-e^{-\kappa_{0}^{2}}\left(D^{+}+\kappa_{0} \sqrt{\pi}\right)\right)\left[U_{1,0}^{\prime \prime}\right]_{-}^{+} \\
-\frac{e^{\kappa_{0}^{2}}}{2 \sqrt{\pi}}\left(\left\{(\sqrt{\pi}-J)\left(\kappa_{0}^{2}-1\right)+\kappa_{0}^{3} D^{-}\right\} D^{+}+\left\{\kappa_{0}^{2}\left[\kappa_{0} D^{+}-J\right]+J\right\} D^{-}\right. \\
\left.\left.-\kappa_{0} e^{-\kappa_{0}^{2}}\left(D^{+}+\kappa_{0} \sqrt{\pi}\right)\right)\left[U_{1,1}^{\prime}\right]_{-}^{+}\right]+O\left(\frac{\epsilon}{\nu^{2}}\right) . \quad(\mathrm{D} 14)
\end{gathered}
$$




\section{E order $\epsilon^{2}$ constant-shear flow}

Some components of $\psi^{(2)}$ are expressed in this subsection through a similar relation to (19) valid at $O\left(\epsilon^{2}\right)$ and with the subsequent relations between $\gamma_{i}^{s}$ and $\beta_{i}^{s}$.

E1 $\partial_{X}^{4} A$

$$
\begin{aligned}
& \phi_{f, 2}^{+}=\frac{\pi \varrho}{2 \bar{\beta}}\left\{\frac{4 \bar{\beta} \eta_{2}^{4}}{9 \varrho_{2}^{2} J_{1}\left(\varrho_{2}\right)}\left[J_{1}\left(\varrho_{2}\right) Y_{1}(\varrho)-Y_{1}\left(\varrho_{2}\right) J_{1}(\varrho)\right]-\frac{4 \bar{\beta} \eta_{2}^{2}}{9 \varrho_{2} J_{1}\left(\varrho_{2}\right)} \frac{\eta^{2}}{\varrho}\left[J_{2}\left(\varrho_{2}\right) Y_{2}(\varrho)-Y_{2}\left(\varrho_{2}\right) J_{2}(\varrho)\right]\right. \\
& +\frac{1}{96 \bar{\beta}^{3}}\left[\frac{\varrho^{6}-\varrho_{2}^{6}}{12 J_{1}\left(\varrho_{2}\right)}\left[Y_{1}(\varrho) J_{1}\left(\varrho_{2}\right)-Y_{1}\left(\varrho_{2}\right) J_{1}(\varrho)\right]+\frac{2}{5} \frac{\varrho^{5}}{J_{1}\left(\varrho_{2}\right)}\left[Y_{1}\left(\varrho_{2}\right) J_{2}(\varrho)-Y_{2}(\varrho) J_{1}\left(\varrho_{2}\right)\right]\right. \\
& -\frac{2}{5} \frac{\varrho_{2}^{5}}{J_{1}\left(\varrho_{2}\right)}\left[Y_{1}(\varrho) J_{2}\left(\varrho_{2}\right)-Y_{2}\left(\varrho_{2}\right) J_{1}(\varrho)\right]+\frac{2}{5} \frac{\varrho^{4}}{J_{1}\left(\varrho_{2}\right)}\left[J_{1}\left(\varrho_{2}\right) Y_{3}(\varrho)-Y_{1}\left(\varrho_{2}\right) J_{3}(\varrho)\right] \\
& \left.\left.-\frac{2}{5} \frac{\varrho_{2}^{4}}{J_{1}\left(\varrho_{2}\right)}\left[J_{1}(\varrho) Y_{3}\left(\varrho_{2}\right)-Y_{1}(\varrho) J_{3}\left(\varrho_{2}\right)\right]\right]\right\} \\
& -\pi \frac{\gamma_{d, 1}^{+}}{J_{1}\left(\varrho_{2}\right)} \frac{\eta^{2}}{3}\left[Y_{1}\left(\varrho_{2}\right) J_{2}(\varrho)-Y_{2}(\varrho) J_{1}\left(\varrho_{2}\right)\right]+\pi \frac{\gamma_{d, 1}^{+}}{J_{1}\left(\varrho_{2}\right)} \frac{\varrho}{\varrho_{2}} \frac{\eta_{2}^{2}}{3}\left[Y_{1}(\varrho) J_{2}\left(\varrho_{2}\right)-Y_{2}\left(\varrho_{2}\right) J_{1}(\varrho)\right]+\gamma_{f, 2}^{+} \phi, \\
& \gamma_{f, 2}^{+}=\beta_{f, 2}^{+}+\gamma_{d, 1}^{+} \varrho_{2}^{3} \frac{J_{2}\left(\varrho_{2}\right)}{24 \bar{\beta}^{2} J_{1}\left(\varrho_{2}\right)}+\frac{\varrho_{2}^{4}}{240 \bar{\beta}^{4}} \frac{J_{3}\left(\varrho_{2}\right)}{J_{1}\left(\varrho_{2}\right)}-\frac{\varrho_{2}^{5}}{240 \bar{\beta}^{4}} \frac{J_{2}\left(\varrho_{2}\right)}{J_{1}\left(\varrho_{2}\right)}+\frac{\varrho_{2}^{6}}{1152 \bar{\beta}^{4}}, \\
& \phi_{f, 2}^{-}=-i \frac{\pi \varrho}{2 \bar{\beta}}\left\{-\frac{4 \bar{\beta} \eta_{1}^{2}}{9 \varrho_{1} J_{1}\left(i \varrho_{1}\right)} \frac{\eta^{2}}{\varrho}\left[J_{2}\left(i \varrho_{1}\right) Y_{2}(i \varrho)-Y_{2}\left(i \varrho_{1}\right) J_{2}(i \varrho)\right]+\frac{4 \bar{\beta} \eta_{1}^{4}}{9 \varrho_{1}^{2} J_{1}\left(i \varrho_{1}\right)}\left[J_{1}\left(i \varrho_{1}\right) Y_{1}(i \varrho)-Y_{1}\left(i \varrho_{1}\right) J_{1}(i \varrho)\right]\right. \\
& +\frac{1}{96 \bar{\beta}^{3}}\left[\frac{\varrho^{6}-\varrho_{1}^{6}}{12 J_{1}\left(i \varrho_{1}\right)}\left[Y_{1}(i \varrho) J_{1}\left(i \varrho_{1}\right)-Y_{1}\left(i \varrho_{1}\right) J_{1}(i \varrho)\right]-\frac{2}{5} i \frac{\varrho^{5}}{J_{1}\left(i \varrho_{1}\right)}\left[Y_{1}\left(i \varrho_{1}\right) J_{2}(i \varrho)-Y_{2}(i \varrho) J_{1}\left(i \varrho_{1}\right)\right]\right. \\
& +\frac{2}{5} i \frac{\varrho_{1}^{5}}{J_{1}\left(i \varrho_{1}\right)}\left[Y_{1}(i \varrho) J_{2}\left(i \varrho_{1}\right)-Y_{2}\left(i \varrho_{1}\right) J_{1}(i \varrho)\right]-\frac{2}{5} \frac{\varrho^{4}}{J_{1}\left(i \varrho_{1}\right)}\left[J_{1}\left(i \varrho_{1}\right) Y_{3}(i \varrho)-Y_{1}\left(i \varrho_{1}\right) J_{3}(i \varrho)\right] \\
& \left.\left.+\frac{2}{5} \frac{\varrho_{1}^{4}}{J_{1}\left(i \varrho_{1}\right)}\left[J_{1}(i \varrho) Y_{3}\left(i \varrho_{1}\right)-Y_{1}(i \varrho) J_{3}\left(i \varrho_{1}\right)\right]\right]\right\} \\
& -\pi \frac{\gamma_{d, 1}^{-}}{J_{1}\left(i \varrho_{1}\right)} \frac{\eta^{2}}{3}\left[Y_{1}\left(i \varrho_{1}\right) J_{2}(i \varrho)-Y_{2}(i \varrho) J_{1}\left(i \varrho_{1}\right)\right]+\pi \frac{\gamma_{d, 1}^{-}}{J_{1}\left(i \varrho_{1}\right)} \frac{\varrho}{\varrho_{1}} \frac{\eta_{1}^{2}}{3}\left[Y_{1}(i \varrho) J_{2}\left(i \varrho_{1}\right)-Y_{2}\left(i \varrho_{1}\right) J_{1}(i \varrho)\right]+\gamma_{f, 2}^{-} \phi, \\
& \gamma_{f, 2}^{-}=\beta_{f, 2}^{-}-i \gamma_{d, 1}^{-} \varrho_{1}^{3} \frac{J_{2}\left(i \varrho_{1}\right)}{24 \bar{\beta}^{2} J_{1}\left(i \varrho_{1}\right)}+\frac{\varrho_{1}^{4}}{240 \bar{\beta}^{4}} \frac{J_{3}\left(i \varrho_{1}\right)}{J_{1}\left(i \varrho_{1}\right)}-i \frac{\varrho_{1}^{5}}{240 \bar{\beta}^{4}} \frac{J_{2}\left(i \varrho_{1}\right)}{J_{1}\left(i \varrho_{1}\right)}-\frac{\varrho_{1}^{6}}{1152 \bar{\beta}^{4}} .
\end{aligned}
$$

E2 $A^{2}$

$$
\begin{array}{r}
\phi_{n}^{(2)}=-2 \bar{R}_{0} \bar{\beta}\left(\mathcal{A}^{+} J_{0}(\varrho)+\mathcal{B}^{+} Y_{0}(\varrho)-\frac{\varrho}{\varrho_{2}} \frac{\mathcal{A}^{+}}{Y_{1}\left(\varrho_{2}\right)}\left[Y_{1}(\varrho) J_{0}\left(\varrho_{2}\right)-J_{1}(\varrho) Y_{0}\left(\varrho_{2}\right)\right]\right) \\
-8 \pi \varrho \bar{\beta}^{2} \int_{\varrho_{2}}^{\varrho} \frac{1}{r^{2}}\left[\mathcal{A}^{+} J_{1}(r)+\mathcal{B}^{+} Y_{1}(r)\right]^{2}\left[J_{1}(\varrho) Y_{1}(r)-J_{1}(r) Y_{1}(\varrho)\right] d r+\gamma_{2, n}^{+} \phi^{+}, \\
\phi_{n}^{(2)-}=2 i \bar{R}_{0} \bar{\beta}\left(\mathcal{A}^{-} J_{0}(i \varrho)+\mathcal{B}^{-} Y_{0}(i \varrho)-\frac{\varrho}{\varrho_{1}} \frac{\mathcal{A}^{-}}{Y_{1}\left(i \varrho_{1}\right)}\left[Y_{1}(i \varrho) J_{0}\left(i \varrho_{1}\right)-J_{1}(i \varrho) Y_{0}(i \varrho 1)\right]\right) \\
+8 \pi \varrho \bar{\beta}^{2} \int_{\varrho}^{\varrho} \frac{1}{r^{2}}\left[\mathcal{A}^{-} J_{1}(i r)+\mathcal{B}^{-} Y_{1}(i r)\right]^{2}\left[J_{1}(i \varrho) Y_{1}(i r)-J_{1}(i r) Y_{1}(i \varrho)\right] d r+\gamma_{2, n}^{-} \phi^{-},
\end{array}
$$




$$
\begin{aligned}
\gamma_{n}^{+}=\beta_{n, 2}^{+}+ & \bar{R}_{0} \bar{\beta}\left(\frac{2}{\varrho_{2}} \frac{J_{0}\left(\varrho_{2}\right)}{J_{1}\left(\varrho_{2}\right)}-2 \gamma+\pi \frac{Y_{1}\left(\varrho_{2}\right)}{J_{1}\left(\varrho_{2}\right)}-\ln \varphi\right) \\
& -2 \pi^{3} \bar{\beta} \bar{\beta} \mathcal{P} \int_{0}^{\varrho_{2}} \frac{\varrho}{r^{2}}\left(\frac{Y_{1}\left(\varrho_{2}\right)}{J_{1}\left(\varrho_{2}\right)} J_{1}(r)-Y_{1}(r)\right)^{2}\left[J_{1}(\varrho) Y_{1}(r)-J_{1}(r) Y_{1}(\varrho)\right] d r+c_{n, 2,1}, \\
\gamma_{2, n}^{-}=\beta_{n, 2}^{-}+ & \bar{R}_{0} \bar{\beta}\left(\frac{2}{i \varrho_{1}} \frac{J_{0}\left(i \varrho_{1}\right)}{J_{2}\left(i \varrho_{1}\right)}-2 \gamma+\pi \frac{Y_{1}\left(i \varrho_{1}\right)}{J_{1}\left(i \varrho_{1}\right)}-\ln -\varphi\right) \\
- & 2 \pi^{3} \bar{\beta} \bar{\beta} \mathcal{P} \int_{0}^{\varrho_{1}} \frac{\varrho}{r^{2}}\left(\frac{Y_{1}\left(i \varrho_{1}\right)}{J_{1}\left(i \varrho_{1}\right)} J_{1}(i r)-Y_{1}(i r)\right)^{2}\left[J_{1}(i \varrho) Y_{1}(i r)-J_{1}(i r) Y_{1}(i \varrho)\right] d r+c_{n, 2,1} .
\end{aligned}
$$

E3 $\mathcal{C}[A]$

$$
\begin{gathered}
\phi_{c}^{(2)+}=2 \overline{\bar{\beta}}\left(\mathcal{A}^{+} J_{0}(\varrho)+\mathcal{B}^{+} Y_{0}(\varrho)-\frac{\mathcal{A}^{+}}{Y_{1}\left(\varrho_{2}\right)} \frac{\varrho}{\varrho_{2}}\left[Y_{1}(\varrho) J_{0}\left(\varrho_{2}\right)-Y_{0}\left(\varrho_{2}\right) J_{1}(\varrho)\right]\right)+\gamma_{c, 2}^{+} \phi^{+}, \\
\phi_{c}^{(2)-}=-2 i \overline{\bar{\beta}}\left(\mathcal{A}^{-} J_{0}(i \varrho)+\mathcal{B}^{-} Y_{0}(i \varrho)+\frac{\mathcal{A}^{-}}{Y_{1}\left(i \varrho_{1}\right)} \frac{\varrho}{\varrho_{1}}\left[Y_{0}\left(i \varrho_{1}\right) J_{1}(i \varrho)-Y_{1}(i \varrho) J_{0}\left(i \varrho_{1}\right)\right]\right)+\gamma_{c, 2}^{-} \phi^{-}, \\
\gamma_{c, 2}^{+}=\beta_{c, 2}^{+}+\overline{\bar{\beta}}\left(1-2 \frac{J_{0}\left(\varrho_{2}\right)}{\varrho_{2} J_{1}\left(\varrho_{2}\right)}\right), \quad \gamma_{c, 2}^{-}=\beta_{c, 2}^{-}+\overline{\bar{\beta}}\left(1-2 \frac{J_{0}\left(i \varrho_{1}\right)}{i \varrho_{1} J_{1}\left(i \varrho_{1}\right)}\right) .
\end{gathered}
$$

\section{E4 Fredholm alternative}

The jumps (55), (58) and (59) are in the constant-shear case:

$$
\begin{gathered}
{\left[\alpha_{n, 2}\right]_{-}^{+}=\frac{32}{9} \bar{\beta} \overline{\bar{\beta}}\left(\frac{1}{\eta_{2} J_{1}^{2}\left(\varrho_{2}\right)}-\frac{1}{\eta_{1} J_{1}^{2}\left(i \varrho_{1}\right)}\right)} \\
-4 \pi^{3} \bar{\beta}^{2} \overline{\bar{\beta}}\left\{i \mathcal{P} \int_{0}^{\varrho_{1}} \frac{1}{\varrho^{2}}\left(\frac{Y_{1}\left(i \varrho_{1}\right)}{J_{1}\left(i \varrho_{1}\right)} J_{1}(i \varrho)-Y_{1}(i \varrho)\right)^{3} d \varrho+\mathcal{P} \int_{0}^{\varrho_{2}} \frac{1}{\varrho^{2}}\left(\frac{Y_{1}\left(\varrho_{2}\right)}{J_{1}\left(\varrho_{2}\right)} J_{1}(\varrho)-Y_{1}(\varrho)\right)^{3} d \varrho\right\}, \quad(\mathrm{E} 25) \\
{\left[\alpha_{f, 2}\right]_{-}^{+}=-\frac{\varrho_{2}^{3}}{72 \bar{\beta}^{3}} \frac{J_{2}\left(\varrho_{2}\right)}{J_{1}\left(\varrho_{2}\right)}-i \frac{\varrho_{1}^{3}}{72 \bar{\beta}^{3}} \frac{J_{2}\left(i \varrho_{1}\right)}{J_{1}\left(i \varrho_{1}\right)}-\frac{1}{120 \bar{\beta}^{3}}\left(\frac{\varrho_{1}^{4}}{J_{1}^{2}\left(i \varrho_{1}\right)}\left(1+\frac{4}{\varrho_{1}^{2}}\right)+16\right)+\frac{1}{120 \bar{\beta}^{3}}\left(\frac{\varrho_{2}^{4}}{J_{1}^{2}\left(\varrho_{2}\right)}\left(1-\frac{4}{\varrho_{2}^{2}}\right)+16\right)} \\
-\frac{\gamma_{d, 1}^{+}}{12 \bar{\beta}}\left(\frac{\varrho_{2}^{2}}{J_{1}^{2}\left(\varrho_{2}\right)}+\frac{\varrho_{1}^{2}}{J_{1}^{2}\left(i \varrho_{1}\right)}\right)+\frac{1}{18 \bar{\beta}}\left(\frac{\eta_{2}^{2}}{\varrho_{2}} \frac{J_{2}\left(\varrho_{2}\right)}{J_{1}\left(\varrho_{2}\right)}-\frac{\eta_{1}^{2}}{i \varrho_{1}} \frac{J_{2}\left(i \varrho_{1}\right)}{J_{1}\left(i \varrho_{1}\right)}\right)\left(\frac{\varrho_{1}^{2}}{J_{1}^{2}\left(i \varrho_{1}\right)}+4\right), \quad(\mathrm{E} 26) \\
{\left[\alpha_{c, 2}\right]_{-}^{+}=\overline{\bar{\beta}}\left(\frac{1}{\eta_{2} J_{1}^{2}\left(\varrho_{2}\right)}-\frac{1}{\eta_{1} J_{1}^{2}\left(i \varrho_{1}\right)}\right) .}
\end{gathered}
$$

